\title{
Variações locais na riqueza florística em duas ecorregiões de caatinga
}

Local changes in floristic richness in two ecorregions of the caatinga

\author{
Grênivel Mota da Costa ${ }^{1,2,4}$, Domingos Cardoso ${ }^{1,3}$, Luciano Paganucci de Queiroz ${ }^{1}$, Abel Augusto Conceição ${ }^{1}$
}

\begin{abstract}
Resumo
A vegetação de caatinga do nordeste brasileiro ocorre principalmente sobre dois tipos de substrato principais: sobre solos derivados do embasamento cristalino pré-cambriano e sobre a cobertura arenosa das bacias sedimentares. Evidências recentes têm demonstrado variações na dinâmica, estrutura e composição florística da caatinga desses dois macroambientes, o que fundamenta a necessidade de estudos com enfoque nas diferenças florísticas entre esses diferentes substratos. O presente estudo florístico das ecorregiões de caatinga, Depressão Sertaneja Meridional e Raso da Catarina, no município de Tucano, Bahia, é de especial interesse porque neste município ocorrem esses dois substratos em escala geográfica local, permitindo ampliar o conhecimento sobre a heterogeneidade do Domínio da Caatinga. Neste trabalho é apresentado um inventário florístico de 14 localidades nestas duas ecorregiões da Caatinga. Em conjunto, as caatingas amostradas apresentaram uma alta riqueza florística em comparação com outras áreas no nordeste do Brasil. Foram coletadas 450 espécies, 288 gêneros e 82 famílias. A maior riqueza em espécies foi das Leguminosae, seguida por Euphorbiaceae. Nas localidades sobre substrato derivado do embasamento cristalino ocorreram 226 espécies, enquanto nas de substrato arenoso ocorreram 284 espécies. A proporção de espécies em comum entre esses dois tipos de substrato foi de apenas $13 \%$. Diferenças em composição florística reforçam a hipótese de duas biotas distintas associadas aos dois principais tipos de substrato, que compõem a vegetação da caatinga.

Palavras-chave: floresta sazonalmente seca, Depressão Sertaneja Meridional, Raso da Catarina, semiárido brasileiro.
\end{abstract}

\begin{abstract}
The caatinga vegetation of northeastern Brazil occurs mainly in Pre-Cambrian, crystalline-derived soils and also on sedimentary-basin-derived sandy soils. Recent evidence has shown variations in the dynamics, structure and floristic composition of the caatinga over these two different edaphic macro-environments, which justifies the need for studies focusing on different substrates. A floristic study of the caatinga ecoregions Depressão Sertaneja Meridional and Raso da Catarina in the municipality of Tucano, Bahia, Brazil, is of special interest because both substrates occur in a local scale, allowing the opportunity to broaden our knowledge on ecological heterogeneity within the Caatinga biome. In the current study we carried out a floristic survey of 14 sites distributed throughout the ecoregions of caatinga in Tucano. We found high species diversity in the caatingas of Tucano when compared to other areas in northeastern Brazil. A total of 450 species, 288 genera, and 82 families were collected. The most species rich families are Leguminosae and Euphorbiaceae. There occur 226 species in the sites on crystalline basement, whereas 284 species were collected in the sandy areas. The ecoregions were only $13 \%$ similar in floristic composition. We found strong differences between the ecoregions with respect to floristic composition and predominant plant habit as proxy for phytophysiognomy. Overall, our results reinforce the hypothesis of two distinct biotas that assembly the caatinga dry woodlands.

Key words: Brazilian semiarid, Depressão Sertaneja Meridional, Raso da Catarina, seasonally dry forest.
\end{abstract}

\footnotetext{
${ }^{1}$ Universidade Estadual de Feira de Santana, PPGBot, Av. Transnordestina, s/n, Novo Horizonte, 44036-900, Feira de Santana, BA, Brasil.

${ }^{2}$ Universidade Federal do Recôncavo da Bahia, Centro de Ciências Agrárias, Ambientais e Biológicas, Rua Rui Barbosa, 710, 44380-000, Cruz das Almas, BA, Brasil.

${ }^{3}$ Universidade Federal da Bahia, Inst. Biologia, Depto. Botânica, Rua Barão de Jeremoabo, s/n, Campus Universitário de Ondina, 40170-115, Salvador, BA, Brasil.

${ }^{4}$ Autor para correspondência: grenivel@gmail.com
} 


\section{Introdução}

A vegetação de caatinga é o maior e um dos mais diversos núcleos das florestas tropicais sazonalmente secas (FTSS), que compõem um bioma neotropical com distribuição disjunta e fortemente marcado pela sazonalidade climática, precipitação anual abaixo de $1200 \mathrm{~mm}$, estrato herbáceo efêmero e alta diversidade e endemismo de Leguminosae (Pennington et al. 2000; Queiroz 2006; Linares-Palomino et al. 2010; Särkinen et al. 2011; Oliveira-Filho et al. 2013). Ocupando uma área de cerca de $850.000 \mathrm{~km}^{2}$, a Caatinga estende-se desde ca. $02^{\circ} 50$ 'S em seu limite norte, nos estados do Ceará e Rio Grande do Norte, até ca. $17^{\circ} 20^{\prime} \mathrm{S}$ em seu limite sul, no norte do estado de Minas Gerais, o que corresponde à maior parte da região semiárida do Nordeste e quase $10 \%$ do território brasileiro (Ab’Saber 1974).

Dentre as mais de $30 \mathrm{mil}$ espécies de angiospermas listadas na flora do Brasil (Forzza et al. 2010), aproximadamente 4500 ocorrem no Domínio da Caatinga. Embora isso represente 15\% das espécies do Brasil, apenas cerca de 1\% da sua vegetação está protegida em unidades de conservação (Leal et al. 2005; Queiroz 2006). Além disso, a conservação da biodiversidade da Caatinga carece de informações básicas, já que aproximadamente $50 \%$ do seu território não têm sido incluídos em programas de inventário de biodiversidade (Santos et al. 2011). Considerando apenas as diversas fitofisionomias da vegetação de caatinga (por exemplo, excluindo os campos rupestres da Chapada Diamantina na Bahia), há pelo menos 318 espécies endêmicas (Giulietti et al. 2002). Apesar de sua importância florística e biogeográfica, a caatinga está entre os tipos de vegetação deciduais mais ameaçados da região Neotropical, sendo alvo de destruição de grandes áreas naturais, o que tem resultado em um crescente processo de fragmentação (Queiroz 2006; Santos et al. 2011; Albuquerque et al. 2012).

As caatingas geralmente têm sido consideradas como uma unidade vegetacional homogênea em estudos que analisam a sua composição florística para inferências biogeográficas e/ou ecológicas em escalas amplas (Pennington et al. 2000; Oliveira-Filho et al. 2006). Entretanto, estudos mostram que há uma forte relação entre o substrato e a variação na vegetação, tanto do ponto de vista fisionômico, quanto florístico e de aspectos morfofuncionais (Queiroz 2006).
A diversidade florística e fitofisionômica da Caatinga (Andrade-Lima 1981; Sampaio et al. 2002; Prado 2003; Queiroz et al. 2006; Santos et al. 2012) corrobora, portanto, ao reconhecimento de diferentes ecorregiões, onde cada uma abriga uma assembleia única de espécies e alto endemismo (Velloso et al. 2002; Queiroz 2006). Provavelmente, essa diversidade de tipos de vegetação responde primariamente às grandes unidades geomorfológicas e, secundariamente, à variação na intensidade do déficit hídrico (Queiroz 2006).

O tipo de solo também pode influenciar na estrutura e dinâmica da vegetação das caatingas e, consequentemente, na sua composição florística (Rodal 1992). Análises de padrões de diversidade, distribuição e endemismos das Leguminosae em áreas de caatingas com fisionomias aparentemente homogêneas têm demonstrado que, sob a denominação de caatinga, provavelmente existam duas biotas historicamente distintas (Queiroz 2006; Cardoso \& Queiroz 2007). Estes autores argumentam que as ecorregiões da Caatinga poderiam ser subdivididas em uma biota associada às superfícies sedimentares arenosas, enquanto a outra associada aos solos geralmente pedregosos derivados de rochas cristalinas pré-cambrianas (Queiroz 2006; Cardoso \& Queiroz 2007; Araújo et al. 2011). De fato, além de apresentar maior densidade de indivíduos por espécie (AndradeLima 1981; Lyra 1982; Rodal 1992; Rocha et al. 2004), a caatinga associada às bacias sedimentares é também mais rica em espécies e endemismos do que a caatinga sobre solos derivados do embasamento cristalino (Queiroz 2006). Características ecológicas marcantes da vegetação de caatinga sobre substrato arenoso incluem, por exemplo, baixa taxa de deciduidade foliar durante a estação seca e muitas espécies com síndromes de dispersão zoocórica (Rodal \& Sampaio 2002; Rocha et al. 2004).

A hipótese de duas biotas distintas relacionadas ao substrato compondo as caatingas foi indicada principalmente a partir de estudos sobre a repartição da flora (Gomes et al. 2006), especialmente, do componente lenhoso (Araújo et al., 2005; Santos et al. 2012), e confirmada por padrões biogeográficos de Leguminosae em diferentes escalas (Queiroz 2006; Cardoso \& Queiroz 2007) e pela distribuição e diversificação do gênero Croton (Euphorbiaceae; Carneiro-Torres 2009). Embora estas sejam as famílias mais diversas e abundantes na caatinga (Queiroz et al. 2006), ainda seria necessário um estudo fitogeográfico mais amplo. 
O objetivo do presente trabalho foi avaliar se a bacia sedimentar e o embasamento cristalino são importantes para distinção da composição e riqueza florísticas na caatinga em escala local. Para isso, foi investigada a composição florística em duas ecorregiões de caatinga (Depressão Sertaneja Meridional e Raso da Catarina) sobre substratos geomorfologicamente distintos foi investigada.

\section{Material e Métodos}

Área de estudo. O município de Tucano

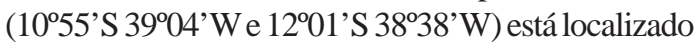
na microrregião de Euclides da Cunha ao nordeste do estado da Bahia. O clima da região é do tipo árido a semiárido, com temperatura média 20,7 a $27,1^{\circ} \mathrm{C}$, precipitação anual de 300 a 800 mm, sem uma estação chuvosa definida, apesar da tendência desta ocorrer entre primavera-verão (SEI 2009). A caatinga é o tipo de vegetação exclusivo no município, embora ela se apresente em diferentes fisionomias e com heterogeneidade florística, a depender das condições locais de solo (Cardoso \& Queiroz 2007). A vegetação sobre solos derivados de rochas cristalinas do préCambriano distribui-se principalmente na parte oeste do município (Fig. 1) e está inserida na ecorregião Depressão Sertaneja Meridional (DS) (Velloso et al. 2002). É nessa área que ocorrem algumas serras com afloramentos graníticos que chegam a alcançar $650 \mathrm{~m}$ de altitude e a fisionomia da vegetação pode variar desde arbustais com abundância de Cactaceae [especialmente Pilosocereus gounellei (F.A.C. Weber ex K. Schum.) Byles \& G.D.Rowley] e Bromeliaceae (Encholirium spectabile Mart. ex Schult.f.), até uma formação arbórea densa, com diversidade e abundância de lianas e até mesmo epífitas. A ecorregião Raso da Catarina (RC) (Velloso et al. 2002) ocorre a leste do município (Fig. 1) e está inserida na bacia do Recôncavo-Tucano-Jatobá, onde são encontradas formações de arenito, com solo em geral menos fértil e clima um pouco mais úmido, com precipitação anual de 800 a 1100 mm (SEI 2009). A caatinga nesta região ocorre principalmente sobre a cobertura arenosa da sub-bacia de Tucano, com formações mais arbustivas.

\section{Levantamento florístico}

Foi realizado a partir de visitas periódicas, entre 2007 e 2013, em 14 fragmentos de caatinga nas duas ecorregiões (Fig. 1), alguns fragmentos foram visitados com maior frequência. Plantas vasculares coletadas previamente nas caatingas em Tucano e depositadas no Herbário da Universidade
Estadual de Feira de Santana (HUEFS) também foram incluídas na listagem florística, desde que providas de informações precisas sobre a localidade de coleta. As espécies foram identificadas a partir de literatura especializada, consulta a taxonomistas e comparação por material depositado em herbário. A classificação e nomenclatura das famílias de angiospermas seguiu o APG III (2009), com exceção de Fabaceae, que é referida como Leguminosae, segundo sugestão de Lewis et al. (2005). As plantas coletadas foram depositadas nos herbários HUEFS e HURB (Herbário do Recôncavo da Bahia). As espécies foram classificadas como ameaçadas de extinção segundo MMA (2008) e acerca do seu endemismo na caatinga (Giulietti et al. 2002; Forzza et al. 2010).

A similaridade entre as duas ecorregiões foi determinada através do índice de Sørensen (Magurran 2013) tanto para a composição florística como para os hábitos (ervas, arbustos, árvores e trepadeiras). Para determinação dos agrupamentos entre os fragmentos amostrados dentro de cada ecorregião, foi utilizada a análise multivariada NMDS (Escalonamento multidimensional não métrico) utilizando o Bray-Curtis como medida de similaridade, processada com uso do programa PAST (Hammer et al. 2001).

\section{Resultados}

Nas duas ecorregiões do município de Tucano foram registradas 450 espécies (448 angiospermas e três pteridófitas), distribuídas em 288 gêneros e 82 famílias (Tab. 1). A família mais rica em espécies foi Leguminosae (97 spp.), seguida por Euphorbiaceae (30), Malpighiaceae (20), Asteraceae (17), Rubiaceae (17), Apocynaceae (16) e Myrtaceae (11). Os gêneros mais diversos foram Croton, Mimosa e Senna (9 spp.), Chamaecrista (8) e Solanum (7). A proporção de hábitos em cada ecorregião, bem como em conjunto, é apresentada na Figura 2. Dentre as espécies coletadas, há o primeiro registro de Pelexia sceptrum (Orchidaceae) na região semiárida do Nordeste do Brasil (C.O.Azevedo \& E.Smidt, comunicação pessoal), além das espécies recém-descritas: Senna bracteosa (Leguminosae-Caesalpinioideae) (Cardoso \& Queiroz 2008), Pseudobombax parvifolium (Malvaceae-Bombacoideae) (Carvalho-Sobrinho \& Queiroz 2010), Justicia angustissima (Acanthaceae) (Côrtes \& Rapini 2011) e Tibouchina caatingae (Melastomataceae) (Freitas et al. 2013). 


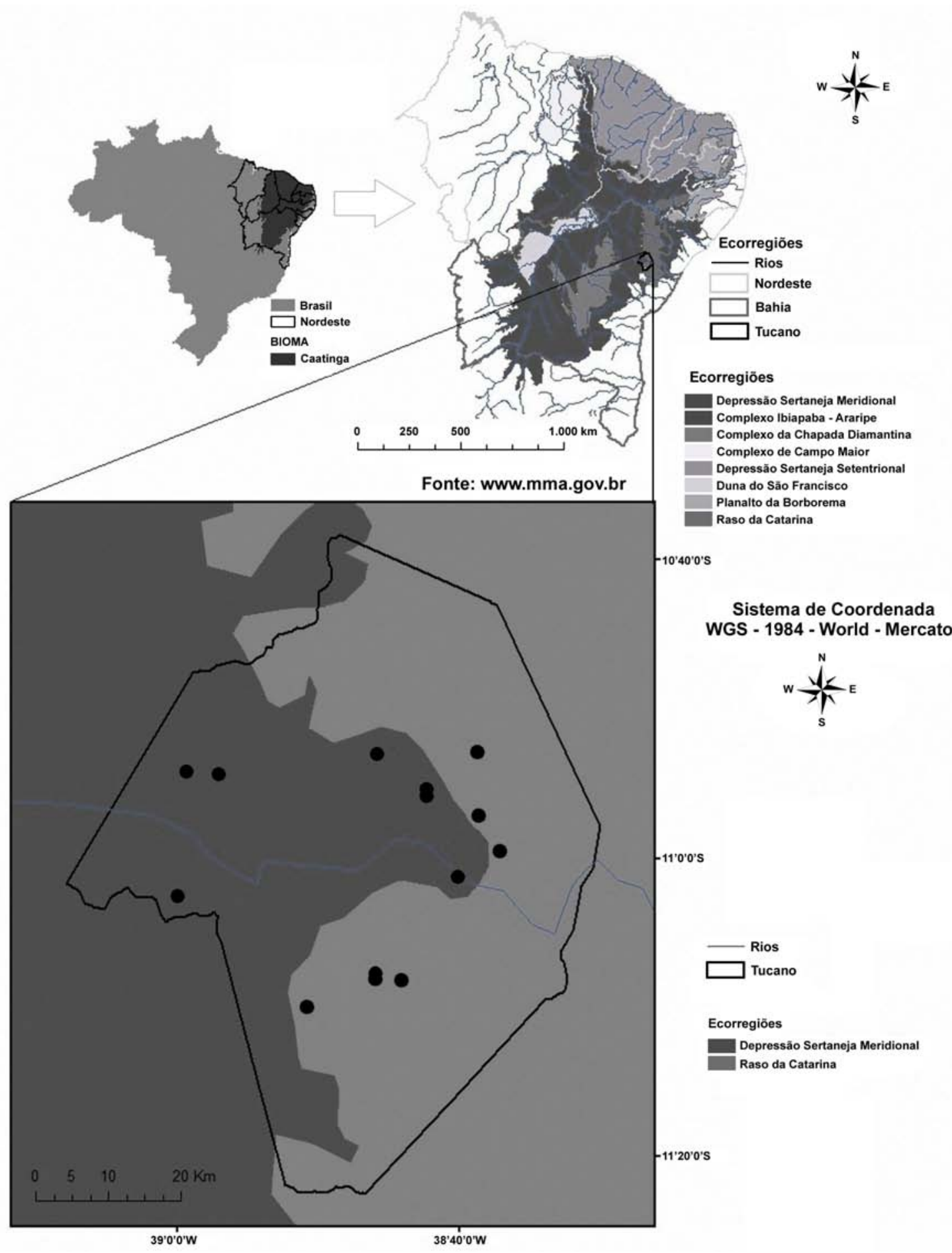

Figura 1 - Pontos de coleta em 14 localidades com fragmentos de caatinga sobre o embasamento cristalino derivado de rochas cristalinas pré-cambrianas e o substrato arenoso originado de bacias sedimentares, município de Tucano, Bahia, Brasil.

Figure 1 - The 14 sampling sites bearing remnants of caatinga on pre-Cambrian crystalline basement and sandy soil from sedimentary basin, municipality of Tucano, Bahia, Brazil. 
Tabela 1 - Lista de espécies ocorrentes em 14 localidades de caatinga sobre bacia sedimentar arenosa (S) e embasamento cristalino (C) no município de Tucano, nordeste da Bahia, Brasil. Siglas para as iniciais dos coletores, hábitos, observações: $\mathrm{AMC}=\mathrm{A} . \mathrm{M}$. de Carvalho; $\mathrm{DC}=\mathrm{D}$. Cardoso; $\mathrm{GC}=\mathrm{G}$. Costa; LCLL = L.C.L.Lima; LPQ = L.P.de Queiroz; HM = H.Maia; MCM = M.C.Machado; MTOM = M.T.O. Menezes; RPO = R.P. Oliveira; arb = arbusto; arv = árvore; epi = epífita; erv = erva; hem = hemiparasita; trep = trepadeira; end = endêmica; $r \mathrm{l}=\mathrm{Red}$ List; ex = exótica. Essa tabela pode ser baixada em formato eletrônico em http://dx.doi.org/10.6084/m9.figshare.1333709. Table 1- List of species occurring in 14 locations of caatinga on sandy sedimentary basin (S) and crystalline basement (C) in the municipality of Tucano, northeastern Bahia, Brazil. Acronym for the initials of collectors, habits, observations: AMC = A.M. de Carvalho; $\mathrm{DC}=$ D.Cardoso; GC = G. Costa; LCLL = L.C.L.Lima; LPQ = L.P. de Queiroz; HM = H.Maia; MCM = M.C.Machado; MTOM = M.T.O. Menezes; RPO = R.P. Oliveira; $\operatorname{arb}=$ shrub; arv = tree; epi = epiphyte; erv = herb; hem = mistletoe; trep = vine; end $=$ endemic; $\mathrm{rl}=$ Red List; ex = exotic. This table is available in electronic format at: http://dx.doi.org/10.6084/m9.figshare.1333709.

\begin{tabular}{|c|c|c|c|c|c|c|}
\hline Família & Espécie & Voucher & $\mathrm{S}$ & $\mathbf{C}$ & Hábito & Obs. \\
\hline \multirow[t]{6}{*}{ Acanthaceae } & Harpochilus neesianus Mart. ex Nees & DC 1196 & $\mathrm{x}$ & & arb & \\
\hline & Justicia aequilabris (Nees) Lindau & DC969, 526 & $\mathrm{x}$ & $\mathrm{x}$ & erv & \\
\hline & Justicia angustissima A.L.A.Côrtes \& Rapini & GC 397 & & $\mathrm{x}$ & erv & end \\
\hline & Justicia thunbergioides (Lindau) Leonard & DC 477 & & $\mathrm{x}$ & erv & \\
\hline & Ruellia asperula (Mart. ex Ness) Lindau & RPO 1432 & & $\mathrm{x}$ & erv & \\
\hline & Thyrsacanthus ramosissimus Moric. & DC 143 & & $\mathrm{x}$ & erv & \\
\hline Alstroemeriaceae & Bomarea sp. & DC 531 & & $\mathrm{x}$ & erv & \\
\hline \multirow[t]{3}{*}{ Amaranthaceae } & Alternanthera ramosissima (Mart.) Chodat & DC 211 & $\mathrm{x}$ & & erv & \\
\hline & Alternanthera tenella Colla & DC 472 & & $\mathrm{x}$ & erv & \\
\hline & Gomphrena demissa Mart. & DC 883, AMC 3883 & $\mathrm{x}$ & $\mathrm{x}$ & erv & \\
\hline Amaryllidaceae & Amaryllidaceae sp. & DC 465 & & $\mathrm{x}$ & erv & \\
\hline \multirow[t]{8}{*}{ Anacardiaceae } & Anacardium occidentale L. & LPQ 3715 & $\mathrm{x}$ & & arv & \\
\hline & Astronium fraxinifolium Schott & DC 940 & $\mathrm{x}$ & & arv & \\
\hline & Astronium graveolens Jacq. & DC 205 & $\mathrm{x}$ & & arv & \\
\hline & Cyrtocarpa caatingae Mitchell \& Daly & DC 525 & & $\mathrm{x}$ & arv & $\mathrm{rl}$ \\
\hline & Schinopsis brasiliensis Engl. & DC 140, 1183 & $\mathrm{x}$ & $\mathrm{x}$ & arv & $\mathrm{rl}$ \\
\hline & Schinus terebinthifolius Raddi & DC 55 & & $\mathrm{x}$ & arv & \\
\hline & $\begin{array}{l}\text { Spondias bahiensis P.Carvalho, van den Berg } \\
\text { \& M.Machado }\end{array}$ & MCM1477 & & $\mathrm{x}$ & arv & \\
\hline & Spondias tuberosa Arruda & DC 2234, 876 & $\mathrm{x}$ & $\mathrm{x}$ & arv & end \\
\hline \multirow[t]{2}{*}{ Annonaceae } & Annona vepretorum Mart. & DC 923 & $\mathrm{x}$ & & arv & end \\
\hline & Oxandra reticulata Maas & GC 469 & $\mathrm{x}$ & & arv & \\
\hline \multirow[t]{11}{*}{ Apocynaceae } & Allamanda blanchetii A.DC. & DC 36 & $\mathrm{x}$ & & arb & end \\
\hline & Aspidosperma pyrifolium Mart. & GC 362, DC 43 & $\mathrm{x}$ & $\mathrm{x}$ & arv & \\
\hline & Blepharodon manicatum (Decne.) Fontella & GC 357 & & $\mathrm{x}$ & trep & $\mathrm{rl}$ \\
\hline & Ditassa arianeae Fontella \& E.A.Schwarz & DC 125 & $\mathrm{x}$ & & trep & $\mathrm{rl}$ \\
\hline & Ditassa capillaris E.Fourn. & DC 895 & $\mathrm{x}$ & & trep & \\
\hline & Ditassa dardanoi T.U.P.Konno \& Wand. & LPQ 9009 & & $\mathrm{x}$ & trep & \\
\hline & Ditassa hastata Decne. & DC 228 & $\mathrm{x}$ & & trep & \\
\hline & Ditassa sp. & DC 3019 & & $\mathrm{x}$ & trep & \\
\hline & Hancornia speciosa Gomez & DC 948 & $\mathrm{x}$ & & arb & \\
\hline & Himatanthus drasticus (Mart.) Plumel & DC 217 & $\mathrm{x}$ & & arb & \\
\hline & Mandevilla funiformis (Vell.) K.Schum. & DC 50 & & $\mathrm{x}$ & trep & \\
\hline
\end{tabular}




\begin{tabular}{|c|c|c|c|c|c|c|}
\hline Família & Espécie & Voucher & $\mathbf{S}$ & C & Hábito & Obs. \\
\hline & $\begin{array}{l}\text { Mandevilla microphylla (Stadelm.) M.F.Sales } \\
\text { \& Kin.-Gouv. }\end{array}$ & DC 974 & $\mathrm{x}$ & & trep & \\
\hline & Marsdenia altissima (Jacq.) Dugand & DC 878 & & $\mathrm{x}$ & arb & \\
\hline & Matelea harleyi Fontella & DC 535 & & $\mathrm{x}$ & trep & \\
\hline & Matelea maritima (Jacq.) Woodson & DC 1323 & & $\mathrm{x}$ & trep & \\
\hline & Matelea nigra (Decne.) Morillo \& Fontella & DC 113 & & $\mathrm{x}$ & trep & \\
\hline \multirow[t]{2}{*}{ Araceae } & Anthurium affine Schott & GC 380 & $\mathrm{x}$ & & erv & \\
\hline & Philodendron leal-costae Mayo \& G.M.Barroso & DC 28 & $\mathrm{x}$ & & erv & \\
\hline \multirow[t]{2}{*}{ Arecaceae } & Arecaceae sp. & GC 873 & $\mathrm{x}$ & & arb & \\
\hline & Syagrus coronata (Mart.) Becc. & - & & $\mathrm{x}$ & arv & \\
\hline Aristolochiaceae & Aristolochia birostris Duchtr. & DC 950 & $\mathrm{x}$ & & trep & \\
\hline \multirow[t]{17}{*}{ Asteraceae } & Aspilia sp. & DC 490 & & $\mathrm{x}$ & erv & \\
\hline & Baccharis cinerea DC. & DC 1339 & & $\mathrm{x}$ & arb & \\
\hline & $\begin{array}{l}\text { Conocliniopsis prasiifolia (DC.) R.M.King } \\
\text { \& H.Rob. }\end{array}$ & GC 334 & & $\mathrm{x}$ & erv & \\
\hline & Cosmos caudatus Kuntze & DC 154 & & $\mathrm{x}$ & erv & \\
\hline & Delilia biflora (L.) Kuntze & DC 1349 & & $\mathrm{x}$ & erv & \\
\hline & $\begin{array}{l}\text { Moquiniastrum oligocephalum (Gardner) G. } \\
\text { Sancho }\end{array}$ & GC 458 & $\mathrm{x}$ & & arb & \\
\hline & $\begin{array}{l}\text { Lepidaploa cotoneaster (Willd.ex Spreng.) } \\
\text { H.Rob. }\end{array}$ & GC 378, AMC3924 & $\mathrm{x}$ & $\mathrm{x}$ & arb & \\
\hline & Lepidaploa fruticosa (L.) H.Rob. & DC 155 & & $\mathrm{x}$ & erv & \\
\hline & Porophyllum ruderale (Jacq.) Cass. & DC 64 & & $\mathrm{x}$ & erv & \\
\hline & Sonchus oleraceus L. & DC 1348 & & $\mathrm{x}$ & erv & \\
\hline & Tilesia baccata (L.f.) Pruski & DC 145, АMC3920 & $\mathrm{x}$ & $\mathrm{x}$ & arb & \\
\hline & $\begin{array}{l}\text { Trixis antimenorrhoea (Schrank) Mart. ex } \\
\text { Baker }\end{array}$ & DC 13, 147 & $\mathrm{x}$ & $\mathrm{x}$ & erv & \\
\hline & Verbesina macrophylla (Cass.) S.F.Blake & DC 1330 & & $\mathrm{x}$ & arb & \\
\hline & Wedelia hookeriana Gardner & GC 468, 367 & $\mathrm{x}$ & $\mathrm{x}$ & arb & \\
\hline & Asteraceae1 & GC 366 & & $\mathrm{x}$ & erv & \\
\hline & Asteraceae2 & DC 512 & & $\mathrm{x}$ & arv & \\
\hline & Asteraceae3 & DC 85 & & $\mathrm{x}$ & erv & \\
\hline Begoniaceae & Begonia saxicola A.DC. & DC 1305 & & $\mathrm{x}$ & erv & \\
\hline \multirow[t]{7}{*}{ Bignoniaceae } & Adenocalymma marginatum (Cham.) DC. & DC 124 & $\mathrm{x}$ & $\mathrm{x}$ & trep & \\
\hline & Anemopaegma laeve DC. & DC 882 & $\mathrm{x}$ & & trep & \\
\hline & Cuspidaria sp. & GC 408 & $\mathrm{x}$ & & trep & \\
\hline & $\begin{array}{l}\text { Fridericia parviflora (Mart. ex DC.) } \\
\text { L.G.Lohmann }\end{array}$ & DC 1184 & $\mathrm{x}$ & & trep & \\
\hline & Fridericia erubescens (DC.) L.G.Lohmann & GC 467 & $\mathrm{x}$ & & trep & \\
\hline & $\begin{array}{l}\text { Handroanthus selachidentatus (A.H.Gentry) } \\
\text { S.O.Grose }\end{array}$ & DC 2236 & & $\mathrm{x}$ & arv & $\mathrm{rl}$ \\
\hline & $\begin{array}{l}\text { Handroanthus impetiginosus (Mart. ex DC.) } \\
\text { Mattos }\end{array}$ & GC 381 & & $\mathrm{x}$ & arv & \\
\hline
\end{tabular}




\begin{tabular}{|c|c|c|c|c|c|c|}
\hline Família & Espécie & Voucher & $\mathbf{S}$ & $\mathbf{C}$ & Hábito & Obs. \\
\hline & Jacaranda jasminoides (Thunb.) Sandwith & DC 927 & $\mathrm{x}$ & & arb & \\
\hline & Pyrostegia venusta (Ker Gawl.) Miers & GC 394 & & $\mathrm{x}$ & trep & \\
\hline \multirow[t]{9}{*}{ Boraginaceae } & Cordia glabrata (Mart.) A.DC. & DC 104, 3032 & $\mathrm{x}$ & $\mathrm{x}$ & arv & \\
\hline & Cordia rufescens A.DC. & DC 190 & $\mathrm{x}$ & & arb & \\
\hline & Cordia trichotoma (Vell.) Arráb. ex Steud. & DC 3021 & & $\mathrm{x}$ & arv & \\
\hline & Heliotropium angiospermum Murray & GC 373 & & $\mathrm{x}$ & erv & \\
\hline & Tournefortia floribunda Kunth & GC 449 & $\mathrm{x}$ & & arb & \\
\hline & Tournefortia rubicunda Salzm. ex A.DC. & DC 899, 552 & $\mathrm{x}$ & $\mathrm{x}$ & arb & \\
\hline & Tournefortia salicifolia A.DC. & DC 984 & $\mathrm{x}$ & & arb & \\
\hline & Varronia globosa Jacq. & LPQ 9013, GC 452 & $\mathrm{x}$ & $\mathrm{x}$ & arb & \\
\hline & Varronia leucocephala (Moric.) J.S.Mill. & DC 77 & & $\mathrm{x}$ & arb & end \\
\hline \multirow[t]{6}{*}{ Bromeliaceae } & Bromelia laciniosa Mart. ex Schult. \& Schult.f. & & & $\mathrm{x}$ & erv & \\
\hline & Cryptanthus bahianus L.B.Sm. & DC 544 & & $\mathrm{x}$ & erv & \\
\hline & $\begin{array}{l}\text { Encholirium spectabile Mart. ex Schult. \& } \\
\text { Schult.f. }\end{array}$ & - & $\mathrm{x}$ & & erv & \\
\hline & Hohenbergia catingae Ule & GC 379 & & $\mathrm{x}$ & erv & \\
\hline & Neoglaziovia variegata (Arruda) Mez & DC 481 & & $\mathrm{x}$ & erv & end \\
\hline & Tillandsia polystachia (L.) L. & DC 3035 & $\mathrm{x}$ & & epi & \\
\hline Burseraceae & Commiphora leptophloeos (Mart.) J.B.Gillett & DC 2226, 528 & $\mathrm{x}$ & $\mathrm{x}$ & arv & \\
\hline \multirow[t]{10}{*}{ Cactaceae } & Arrojadoa rhodantha (Gürke) Britton \& Rose & DC 47 & & $\mathrm{x}$ & arb & end \\
\hline & Cereus jamacaru DC. & - & $\mathrm{x}$ & $\mathrm{x}$ & arv & \\
\hline & Harrisia adscendens (Gürke) Britton \& Rose & - & & $\mathrm{x}$ & arb & end \\
\hline & $\begin{array}{l}\text { Melocactus zehntneri (Britton \& Rose) } \\
\text { Luetzelb. }\end{array}$ & DC 2224 & $\mathrm{x}$ & & arb & \\
\hline & $\begin{array}{l}\text { Pilosocereus catingicola (Gürke) Byles \& } \\
\text { Rowley }\end{array}$ & DC 976 & $\mathrm{x}$ & & arb & \\
\hline & $\begin{array}{l}\text { Pilosocereus tuberculatus (Werderm.) Byles } \\
\text { \& Rowley }\end{array}$ & DC 981 & $\mathrm{x}$ & & arv & end \\
\hline & $\begin{array}{l}\text { Pilosocereus gounellei (F.A.C.Weber) Byles } \\
\text { \& Rowley }\end{array}$ & DC 62 & & $\mathrm{x}$ & arb & \\
\hline & $\begin{array}{l}\text { Tacinga inamoena (K.Schum.) N.P.Taylor \& } \\
\text { Stuppy }\end{array}$ & DC 890 & $\mathrm{x}$ & & arb & \\
\hline & $\begin{array}{l}\text { Tacinga subcylindrica M.Machado \& } \\
\text { N.P.Taylor stat. nov. ined. }\end{array}$ & МТОМ 329 & $\mathrm{x}$ & & erv & \\
\hline & $\begin{array}{l}\text { Tacinga palmadora (Britton \& Rose) } \\
\text { N.P.Taylor \& Stuppy }\end{array}$ & GC 386 & & $\mathrm{x}$ & arb & end \\
\hline Cannaceae & Canna indica $\mathrm{L}$. & DC 87 & & $\mathrm{x}$ & erv & \\
\hline \multirow[t]{4}{*}{ Capparaceae } & Colicodendron yco Mart. & GC 872, DC 115 & $\mathrm{x}$ & $\mathrm{x}$ & arb & end \\
\hline & Crataeva tapia L. & - & & $\mathrm{x}$ & arv & \\
\hline & Cynophalla flexuosa (L.) J.Presl. & DC 171, 105 & $\mathrm{x}$ & $\mathrm{x}$ & arb & \\
\hline & $\begin{array}{l}\text { Neocalyptrocalyx longifolium (Mart.) Cornejo } \\
\text { \& Iltis }\end{array}$ & DC 7,114 & $\mathrm{x}$ & $\mathrm{x}$ & arb & \\
\hline Caricaceae & Jacaratia corumbensis Kuntze & DC 884, 2349 & $\mathrm{x}$ & & arb & \\
\hline Celastraceae & Maytenus patens Reissek & GC 383 & & $\mathrm{x}$ & arv & \\
\hline
\end{tabular}




\begin{tabular}{|c|c|c|c|c|c|c|}
\hline Família & Espécie & Voucher & $\mathbf{S}$ & C & Hábito & Obs. \\
\hline & Maytenus obtusifolia Mart. & DC 215 & $\mathrm{x}$ & & arv & \\
\hline & Maytenus rigida Mart. & GC 433, 455 & $\mathrm{x}$ & $\mathrm{x}$ & arv & \\
\hline Chrysobalanaceae & Hirtella racemosa Lam. & DC 183 & $\mathrm{x}$ & & arb & \\
\hline Cleomaceae & Cleome sp. & DC 506 & & $\mathrm{x}$ & erv & \\
\hline \multirow[t]{2}{*}{ Combretaceae } & Combretum monetaria Mart. & LQ 9021 & & $\mathrm{x}$ & arv & end \\
\hline & Combretum fruticosum (Loefl.) Stuntz & DC 2060 & & $\mathrm{x}$ & arv & \\
\hline \multirow[t]{2}{*}{ Commelinaceae } & Callisia repens (Jacq.) L. & GC 346 & & $\mathrm{x}$ & erv & \\
\hline & Commelina sp. & DC 1307 & & $\mathrm{x}$ & erv & \\
\hline \multirow[t]{8}{*}{ Convolvulaceae } & Evolvulus elegans Moric. & AMC 3908 & $\mathrm{x}$ & & erv & \\
\hline & Evolvulus frankenioides Moric. & DC 222 & & $\mathrm{x}$ & erv & \\
\hline & Ipomoea incarnata (Vahl) Choisy & DC 163 & & $\mathrm{x}$ & trep & \\
\hline & Ipomoea nil (L.) Roth & DC 67 & & $\mathrm{x}$ & trep & \\
\hline & $\begin{array}{l}\text { Jacquemontia heterantha (Nees \& Mart.) } \\
\text { Hallier f. }\end{array}$ & DC 70 & & $\mathrm{x}$ & trep & \\
\hline & Jacquemontia nodiflora (Desr.) G.Don & DC 1317 & & $\mathrm{x}$ & trep & \\
\hline & Merremia aegyptia (L.) Urb. & DC 103 & & $\mathrm{x}$ & trep & \\
\hline & Operculina macrocarpa (L.) Urb. & DC 1355 & & $\mathrm{x}$ & trep & \\
\hline \multirow[t]{3}{*}{ Cucurbitaceae } & Apodanthera congestiflora Cogn. & DC 470 & & $\mathrm{x}$ & trep & end \\
\hline & Apodanthera glaziovii Cogn. & DC 142 & & $\mathrm{x}$ & trep & end \\
\hline & Cayaponia tayuya (Vell.) Cogn. & DC 1314 & & $\mathrm{x}$ & trep & \\
\hline \multirow[t]{5}{*}{ Cyperaceae } & $\begin{array}{l}\text { Bulbostylis lagoensis (Boeckeler) Prata \& } \\
\text { M.G.López }\end{array}$ & GC 411b & & $\mathrm{x}$ & erv & \\
\hline & Bulbostylis sp. & GC 391 & & $\mathrm{x}$ & erv & \\
\hline & Cyperus amabilis Vahl & GC 411a & & $\mathrm{x}$ & erv & \\
\hline & Cyperus sp. & GC 395 & & $\mathrm{x}$ & erv & \\
\hline & Fimbristylis cymosa (Lam.) R.Br. & AMC 3876 & & $\mathrm{x}$ & erv & \\
\hline Dilleniaceae & Curatella americana $\mathrm{L}$. & DC 187 & $\mathrm{x}$ & & erv & \\
\hline \multirow[t]{4}{*}{ Dioscoriaceae } & Dioscorea dodecaneura Vell. & DC 1313 & & $\mathrm{x}$ & trep & \\
\hline & Dioscorea ovata Vell. & DC 1304 & & $\mathrm{x}$ & trep & \\
\hline & $\begin{array}{l}\text { Dioscorea polygonoides Humb. \& Bonpl. ex } \\
\text { Willd. }\end{array}$ & GC 331 & & $\mathrm{x}$ & trep & \\
\hline & Dioscorea sp. & DC 3024 & & $\mathrm{x}$ & trep & \\
\hline Eriocaulaceae & Paepalanthus myocephalus Mart. & DC 2708 & $\mathrm{x}$ & & erv & \\
\hline \multirow[t]{3}{*}{ Erythroxylaceae } & Erythroxylum caatingae Plowman & GC 412, DC 1192 & $\mathrm{x}$ & $\mathrm{x}$ & arv & \\
\hline & Erythroxylum maracasense Plowman & DC 968 & $\mathrm{x}$ & & $\operatorname{arv}$ & \\
\hline & Erythroxylum suberosum A.St.-Hil. & AMC 3922, DC 181 & $\mathrm{x}$ & $\mathrm{x}$ & arv & \\
\hline \multirow[t]{7}{*}{ Euphorbiaceae } & Acalypha brasiliensis Mull.Arg. & DC 915, GC 393 & $\mathrm{x}$ & $\mathrm{x}$ & arb & \\
\hline & Cnidoscolus adenochlamys Fern.Casas & DC 156 & & $\mathrm{x}$ & arb & \\
\hline & Cnidoscolus pubescens Pohl & DC 2250, 164 & $\mathrm{x}$ & $\mathrm{x}$ & arb & \\
\hline & Cnidoscolus quercifolius Pohl & DC 886, AMC 3856 & $\mathrm{x}$ & $\mathrm{x}$ & arb & \\
\hline & Cnidoscolus urens (L.) Arthur & DC 112, GC 871 & $\mathrm{x}$ & $\mathrm{x}$ & arb & \\
\hline & Croton argyrophyllus Kunth & GC 459 & $\mathrm{x}$ & & arb & \\
\hline & Croton heliotropiifolius Kunth & DC 119 & & $\mathrm{x}$ & arb & \\
\hline
\end{tabular}




\begin{tabular}{|c|c|c|c|c|c|c|}
\hline Família & Espécie & Voucher & $\mathbf{S}$ & $\mathbf{C}$ & Hábito & Obs. \\
\hline & Croton adamantinus Müll.Arg. & DC 2245 & $\mathrm{x}$ & & arb & \\
\hline & Croton echioides Baill. & DC 929 & $\mathrm{x}$ & & arb & \\
\hline & Croton grewioides Baill. & DC 906, 545 & $\mathrm{x}$ & $\mathrm{x}$ & artb & \\
\hline & Croton rudolphianus Müll.Arg. & DC 902 & $\mathrm{x}$ & & arb & \\
\hline & Croton tetradenius Baill. & GC 430 & & $\mathrm{x}$ & arb & \\
\hline & Croton urticifolius Lam. & DC 498 & & $\mathrm{x}$ & arb & \\
\hline & Croton virgultosus Müll.Arg. & DC 1194 & $\mathrm{x}$ & & arb & \\
\hline & Dalechampia brasiliensis Lam. & DC 901 & $\mathrm{x}$ & & trep & \\
\hline & $\begin{array}{l}\text { Ditaxis desertorum (Müll.Arg.) Pax \& } \\
\text { K.Hoffm. }\end{array}$ & DC 494 & & $\mathrm{x}$ & erv & end \\
\hline & Euphorbia insulana Vell. & DC 931 & $\mathrm{x}$ & & erv & \\
\hline & Euphorbia phosphorea Mart. & DC 885 & & $\mathrm{x}$ & erv & \\
\hline & Euphorbia prostrata Aiton & DC 911 & $\mathrm{x}$ & & arb & \\
\hline & Jatropha martiusii Baill. & DC 879 & & $\mathrm{x}$ & arb & \\
\hline & Jatropha mollissima (Pohl) Baill. & GC 358, DC 919 & $\mathrm{x}$ & $\mathrm{x}$ & arb & \\
\hline & Jatropha mutabilis (Pohl) Baill. & GC 869 & & $\mathrm{x}$ & arb & end \\
\hline & Jatropha ribifolia (Pohl) Baill. & DC 135 & & $\mathrm{x}$ & arb & \\
\hline & $\begin{array}{l}\text { Manihot allemi P.Carvalho \& M.Martins sp. } \\
\text { nov. ined. }\end{array}$ & GC 852 & $\mathrm{x}$ & & arb & end \\
\hline & Manihot carthagenensis (Jacq.) Müll.Arg. & AMC 3935 & & $\mathrm{x}$ & arv & \\
\hline & $\begin{array}{l}\text { Manihot elongata P.Carvalho \& M.Martins } \\
\text { sp. nov. ined. }\end{array}$ & GC 870 & & $\mathrm{X}$ & arb & \\
\hline & Maprounea guianensis Aubl. & DC 1301 & & $\mathrm{x}$ & $\operatorname{arv}$ & \\
\hline & Sapium glandulosum (L.) Morong & DC 503, 1193 & $\mathrm{x}$ & $\mathrm{x}$ & arb & \\
\hline & Sebastiania sp. & LQ 9028 & & $\mathrm{x}$ & arb & \\
\hline & Tragia sp. & DC 541 & $\mathrm{x}$ & & trep & \\
\hline Gesneriaceae & $\begin{array}{l}\text { Sinningia nordestina Chautems, Baracho \& } \\
\text { J.A.Siqueira }\end{array}$ & DC 2704 & $\mathrm{x}$ & & erv & \\
\hline Krameriaceae & Krameria tomentosa A.St.-Hil. & DC 192 & $\mathrm{x}$ & & arb & \\
\hline \multirow[t]{7}{*}{ Lamiaceae } & $\begin{array}{l}\text { Eplingiella fruticosa (Salzm. ex Benth.) Harley } \\
\text { \& J.F.B.Pastore }\end{array}$ & DC 2712 & $\mathrm{x}$ & & arb & \\
\hline & Eriope tumidicaulis Harley & DC 159 & & $\mathrm{x}$ & erv & \\
\hline & Leonotis nepetifolia (L.) R.Br. & DC 1342 & & $\mathrm{x}$ & arb & ex \\
\hline & $\begin{array}{l}\text { Medusantha martiusii (Benth.) Harley \& } \\
\text { J.F.B.Pastore }\end{array}$ & GC 403 & & $\mathrm{x}$ & arb & \\
\hline & $\begin{array}{l}\text { Mesosphaerum sidifolium (L’Hérit.) Harley \& } \\
\text { J.F.B.Pastore }\end{array}$ & DC 27, GC 369 & $\mathrm{x}$ & $\mathrm{x}$ & erv & \\
\hline & Ocimum campechianum Mill. & LPQ 9027 & & $\mathrm{x}$ & erv & \\
\hline & Rhaphiodon echinus Schauer & DC 35 & $\mathrm{x}$ & & erv & \\
\hline \multirow[t]{4}{*}{ Leguminosae } & Aeschynomene martii Benth. & DC 2242 & $\mathrm{x}$ & & arb & end \\
\hline & Aeschynomene mollicula Kunth & DC 466, 91 & & $\mathrm{x}$ & erv & \\
\hline & Aeschynomene viscidula Michx. & DC 223 & $\mathrm{x}$ & & arb & \\
\hline & Amburana cearensis (Allemão) A.C.Sm. & DC 2056 & & $\mathrm{x}$ & arv & \\
\hline
\end{tabular}




\begin{tabular}{|c|c|c|c|c|c|c|}
\hline Família & Espécie & Voucher & $\mathbf{S}$ & C & Hábito & Obs. \\
\hline & Anadenanthera colubrina (Vell.) Brenan & GC 359 & & $\mathrm{x}$ & arv & \\
\hline & $\begin{array}{l}\text { Ancistrotropis peduncularis (Fawcett \& } \\
\text { Rendle) A.Delgado }\end{array}$ & DC 89 & & $\mathrm{x}$ & trep & \\
\hline & Andira humilis Mart.ex Benth. & DC 985 & $\mathrm{x}$ & & erv & \\
\hline & Bauhinia aculeata L. & DC 485 & & $\mathrm{x}$ & arb & \\
\hline & Bauhinia coriifolia L.P.Queiroz sp. nov. ined. & DC 219 & $\mathrm{x}$ & & arb & \\
\hline & Bauhinia cheilantha (Bong.) Steud. & GC 363 & & $\mathrm{x}$ & arb & \\
\hline & Bauhinia dumosa Benth. & GC 418 & & $\mathrm{x}$ & arb & \\
\hline & Bauhinia subclavata Benth. & GC 460, DC 158 & $\mathrm{x}$ & $\mathrm{x}$ & arb & \\
\hline & Bowdichia virgilioides Kunth & LQ 3711 & $\mathrm{x}$ & & arv & \\
\hline & Calliandra aeschynomenoides Benth. & DC 966, 959 & $\mathrm{x}$ & & arb & end \\
\hline & Canavalia brasiliensis Mart. ex Benth. & DC 116 & & $\mathrm{x}$ & trep & \\
\hline & Centrosema arenarium Benth. & DC 930 & $\mathrm{x}$ & & trep & \\
\hline & Centrosema pubescens Benth. & DC 896 & $\mathrm{x}$ & & trep & \\
\hline & Centrosema virginianum (L.) Benth. & GC 370 & & $\mathrm{x}$ & trep & \\
\hline & Chaetocalyx scandens (L.) Urb. & DC 11, LPQ 9005 & $\mathrm{x}$ & $\mathrm{x}$ & trep & \\
\hline & Chaetocalyx blanchetiana (Benth.) Rudd & DC 53 & & $\mathrm{x}$ & trep & \\
\hline & $\begin{array}{l}\text { Chamaecrista repens (Vogel) H.S.Irwin \& } \\
\text { Barneby }\end{array}$ & DC 889 & & $\mathrm{x}$ & erv & \\
\hline & $\begin{array}{l}\text { Chamaecrista amiciella (H.S.Irwin \& Barneby) } \\
\text { H.S.Irwin \& Barneby }\end{array}$ & DC 209 & $\mathrm{x}$ & & erv & \\
\hline & $\begin{array}{l}\text { Chamaecrista barbata (Nees \& Mart.) } \\
\text { H.S.Irwin \& Barneby }\end{array}$ & DC 951 & $\mathrm{x}$ & & arb & \\
\hline & $\begin{array}{l}\text { Chamaecrista belemii (H.S.Irwin \& Barneby) } \\
\text { H.S.Irwin \& Barneby }\end{array}$ & DC 141, 2248 & $\mathrm{x}$ & $\mathrm{x}$ & arb & end/rl \\
\hline & $\begin{array}{l}\text { Chamaecrista brevicalyx (Benth.) H.S.Irwin } \\
\text { \& Barneby }\end{array}$ & DC 1189 & $\mathrm{x}$ & & arb & \\
\hline & Chamaecrista flexuosa (L.) Greene & DC 1347 & & $\mathrm{x}$ & erv & \\
\hline & Chamaecrista serpens (L.) Greene & DC 909 & $\mathrm{x}$ & & erv & \\
\hline & $\begin{array}{l}\text { Chamaecrista swainsonii (Benth.) H.S.Irwin } \\
\text { \& Barneby }\end{array}$ & DC 206 & $\mathrm{x}$ & $\mathrm{x}$ & erv & \\
\hline & Chloroleucon foliolosum (Benth.) G.P.Lewis & GC 355 & & $\mathrm{x}$ & arv & \\
\hline & $\begin{array}{l}\text { Copaifera arenicola (Ducke) J.A.S.Costa \& } \\
\text { L.P.Queiroz }\end{array}$ & LPQ 13677, DC 26 & $\mathrm{x}$ & & $\operatorname{arv}$ & \\
\hline & Copaifera duckei Dwyer & DC 982 & $\mathrm{x}$ & & arv & \\
\hline & Crotalaria holosericea Nees \& Mart. & GC 352 & & $\mathrm{x}$ & arb & end \\
\hline & $\begin{array}{l}\text { Dahlstedtia araripensis (Benth.) M.J. Silva \& } \\
\text { A.M.G.Azevedo }\end{array}$ & DC 188 & $\mathrm{x}$ & & $\operatorname{arv}$ & \\
\hline & Dalbergia cearensis Ducke & GC 410 & & $\mathrm{x}$ & $\operatorname{arv}$ & \\
\hline & Desmanthus pernambucanus (L.) Thell. & DC 71 & & $\mathrm{x}$ & erv & \\
\hline & Desmodium incanum DC. & LPQ 9031 & & $\mathrm{x}$ & erv & \\
\hline & Dioclea grandiflora Mart. ex Benth. & DC 1, 492 & $\mathrm{x}$ & $\mathrm{x}$ & trep & \\
\hline & Erythrina velutina Willd. & DC 881 & & $\mathrm{x}$ & arv & \\
\hline & Galactia remansoana Harms & DC 964 & $\mathrm{x}$ & & erv & \\
\hline
\end{tabular}




\begin{tabular}{|c|c|c|c|c|c|c|}
\hline Família & Espécie & Voucher & $\mathrm{S}$ & $\mathrm{C}$ & Hábito & Obs. \\
\hline & Geoffroea spinosa Jacq. & LPQ 9000 & & $\mathrm{x}$ & arv & \\
\hline & Goniorrhachis marginata Taub. & DC 510, 26 & $\mathrm{x}$ & $\mathrm{x}$ & arv & \\
\hline & Hymenaea martiana Hayne & DC 922 & $\mathrm{x}$ & & arv & \\
\hline & Indigofera microcarpa Desv. & AMC 3881 & $\mathrm{x}$ & & erv & \\
\hline & Indigofera suffruticosa Mill. & GC 398 & & $\mathrm{x}$ & arb & \\
\hline & Inga laurina (Sw.) Willd. & DC 933 & $\mathrm{x}$ & & arv & \\
\hline & Libidibia ferrea (Mart. ex Tul.) L.P.Queiroz & GC 361 & & $\mathrm{x}$ & arv & \\
\hline & Lonchocarpus sericeus (Poir) Kunth ex DC. & DC 2058 & & $\mathrm{x}$ & arv & \\
\hline & Macroptilium lathyroides (L.) Urb. & GC 335 & & $\mathrm{x}$ & trep & \\
\hline & Mimosa acutistipula (Mart.) Benth. & DC 157 & & $\mathrm{x}$ & arb & \\
\hline & Mimosa arenosa (Willd.) Poir. & DC 128 & & $\mathrm{x}$ & arb & \\
\hline & Mimosa brevipinna Benth. & DC 194 & $\mathrm{x}$ & & erv & end \\
\hline & Mimosa lewisii Barneby & GC 457 & $\mathrm{x}$ & & arb & \\
\hline & Mimosa misera Benth. & DC 2723 & $\mathrm{x}$ & & erv & \\
\hline & Mimosa ophthalmocentra Mart. ex Benth. & AMC 3923 & $\mathrm{x}$ & & arv & end \\
\hline & Mimosa quadrivalvis L. & LCLL 182 & $\mathrm{x}$ & & erv & \\
\hline & Mimosa sensitiva $\mathrm{L}$. & DC 130 & $\mathrm{x}$ & & erv & \\
\hline & Mimosa tenuiflora (Willd.) Poir. & GC360 & & $\mathrm{x}$ & arb & \\
\hline & $\begin{array}{l}\text { Parapiptadenia zehntneri (Harms) M.P.Lima } \\
\text { \& H.C.Lima }\end{array}$ & DC 3011 & & $\mathrm{x}$ & arv & \\
\hline & Parkia platycephala Benth. & DC 560 & $\mathrm{x}$ & & arv & \\
\hline & Parkinsonia aculeata L. & DC 82 & & $\mathrm{x}$ & arb & \\
\hline & Peltogyne pauciflora Benth. & DC 3038, GC 429 & $\mathrm{x}$ & $\mathrm{x}$ & arv & \\
\hline & Periandra mediterranea (Vell.) Taub. & DC 184, GC 868 & $\mathrm{x}$ & & arb & \\
\hline & Piptadenia stipulacea (Benth.) Ducke & LPQ 3115 & & $\mathrm{x}$ & arb & \\
\hline & Piptadenia viridiflora (Kunth) Benth. & DC 550 & & $\mathrm{x}$ & arv & \\
\hline & Pithecellobium diversifolium Benth. & AMC 3882 & $\mathrm{x}$ & & arb & \\
\hline & $\begin{array}{l}\text { Pityrocarpa moniliformis (Benth.) Luckow \& } \\
\text { R.W.Jobson }\end{array}$ & GC 450 & $\mathrm{x}$ & & arb & \\
\hline & Platymiscium floribundum Vogel & DC 3022 & & $\mathrm{x}$ & arv & \\
\hline & Poecilanthe ulei (Harms) Arroyo \& Rudd & DC 478, DC 891 & $\mathrm{x}$ & $\mathrm{x}$ & arv & \\
\hline & Poeppigia procera C.Presl & HM 19, GC 461 & $\mathrm{x}$ & & arv & \\
\hline & $\begin{array}{l}\text { Poincianella microphylla (Mart.ex G.Don) } \\
\text { L.P.Queiroz }\end{array}$ & GC 447 & $\mathrm{x}$ & & arb & end \\
\hline & Poincianella pyramidalis (Tul.) L.P.Queiroz & GC 407, DC 107 & $\mathrm{x}$ & $\mathrm{x}$ & arb & \\
\hline & Poiretia punctata (Willd.) Desv. & GC 377 & & $\mathrm{x}$ & trep & \\
\hline & Rhynchosia phaseoloides (Sw.) DC. & GC 375 & & $\mathrm{x}$ & erv & \\
\hline & $\begin{array}{l}\text { Senegalia piauhiensis (Benth.) Seigler \& } \\
\text { Ebinger }\end{array}$ & LPQ 13675 & $\mathrm{x}$ & & arb & \\
\hline & Senegalia bahiensis (Benth.) Seigler \& Ebinger & GC 400 & $\mathrm{x}$ & $\mathrm{x}$ & arv & \\
\hline & $\begin{array}{l}\text { Senegalia langsdorffii (Benth.) Seigler \& } \\
\text { Ebinger }\end{array}$ & DC 550 & & $\mathrm{x}$ & arb & \\
\hline
\end{tabular}




\begin{tabular}{|c|c|c|c|c|c|c|}
\hline Família & Espécie & Voucher & $\mathbf{S}$ & $\mathbf{C}$ & Hábito & Obs. \\
\hline & $\begin{array}{l}\text { Senna acuruensis (Benth.) H.S.Irwin \& } \\
\text { Barneby }\end{array}$ & DC 120,DC 949 & $\mathrm{x}$ & $\mathrm{x}$ & arb & end \\
\hline & Senna alata (L.) Roxb. & DC 979 & & $\mathrm{x}$ & arb & \\
\hline & $\begin{array}{l}\text { Senna bracteosa D.B.O.S.Cardoso \& } \\
\text { L.P.Queiroz }\end{array}$ & GC 339 & & $\mathrm{x}$ & arb & end \\
\hline & $\begin{array}{l}\text { Senna macranthera (DC. ex Collad.) H.S.Irwin } \\
\text { \& Barneby }\end{array}$ & DC 72 & & $\mathrm{x}$ & arb & \\
\hline & Senna occidentalis (L.) Link & AMC 3869 & & $\mathrm{x}$ & erv & \\
\hline & Senna pendula (Willd.) H.S.Irwin \& Barneby & GC 389 & & $\mathrm{x}$ & arb & \\
\hline & Senna splendida (Vogel) H.S.Irwin \& Barneby & DC 32 & $\mathrm{x}$ & & arb & \\
\hline & Senna rizzinii H.S.Irwin \& Barneby & GC 446, DC 118 & $\mathrm{x}$ & $\mathrm{x}$ & arb & \\
\hline & Senna uniflora (Mill.) H.S.Irwin \& Barneby & DC 61 & & $\mathrm{x}$ & erv & \\
\hline & Sesbania exasperata Kunth & DC 2059 & & $\mathrm{x}$ & arb & \\
\hline & $\begin{array}{l}\text { Stylosanthes pilosa M.B.Ferreira \& Sousa } \\
\text { Costa }\end{array}$ & DC 197 & $\mathrm{x}$ & & erv & \\
\hline & Stylosanthes scabra Vogel & DC 83 & & $\mathrm{x}$ & erv & \\
\hline & Stylosanthes viscosa (L.) Sw. & DC 912 & $\mathrm{x}$ & & erv & \\
\hline & Swartzia apetala Raddi & DC 989 & $\mathrm{x}$ & & erv & \\
\hline & Tephrosia purpurea (L.) Pers. & DC 965 & $\mathrm{x}$ & & erv & \\
\hline & Trischidium molle (Benth.) H.E.Ireland & GC 419,454 & $\mathrm{x}$ & & arb & \\
\hline & Vachellia farnesiana (L.) Wight \& Arn. & AMC 3857 & & $\mathrm{x}$ & arv & \\
\hline & Zornia brasiliensis Vogel. & DC 2240 & & $\mathrm{x}$ & erv & \\
\hline & Zornia echinocarpa (Moric.ex Meisn.) Benth. & DC 962 & $\mathrm{x}$ & & arb & end \\
\hline & Zornia myriadena Benth. & DC 534 & & $\mathrm{x}$ & erv & \\
\hline & Zornia reticulata $\mathrm{Sm}$. & DC 94 & & $\mathrm{x}$ & erv & \\
\hline Loasaceae & Aosa rupestris (Gardner) Weigend & DC 58 & $\mathrm{x}$ & & erv & \\
\hline Loranthaceae & Struthanthus syringifolius (Mart.) Mart. & LPQ 3706 & $\mathrm{x}$ & & hem & \\
\hline \multirow[t]{4}{*}{ Lythraceae } & Cuphea brachiata Koehne & DC 947 & $\mathrm{x}$ & & erv & \\
\hline & Cuphea pulchra Moric. & DC 954 & $\mathrm{x}$ & & eer & \\
\hline & Lafoensia glyptocarpa Koehne & GC 427 & & $\mathrm{x}$ & arv & \\
\hline & Pleurophora anomala A.St.-Hil. & GC 417 & & $\mathrm{x}$ & erv & \\
\hline \multirow[t]{12}{*}{ Malpighiaceae } & Amorimia rigida (A.Juss.) W.R.Anderson & DC 40 & & $\mathrm{x}$ & trep & \\
\hline & Banisteriopsis stellaris (Griseb.) B.Gates & DC 3037 & $\mathrm{x}$ & & arb & \\
\hline & Byrsonima gardnerana A.Juss. & DC 920 & $\mathrm{x}$ & & arb & \\
\hline & Byrsonima coccolobifolia Kunth & GC 462 & $\mathrm{x}$ & & arb & \\
\hline & Byrsonima dealbata Griseb. & DC 182 & $\mathrm{x}$ & & arb & \\
\hline & Byrsonima verbascifolia (L.) DC. & LPQ 3713 & $\mathrm{x}$ & & arb & \\
\hline & Carolus chasei (W.R.Anderson) W.R.Anderson & GC 422 & $\mathrm{x}$ & & trep & \\
\hline & Galphimia brasiliensis (L.) A.Juss. & DC 916, 349 & $\mathrm{x}$ & $\mathrm{x}$ & arb & \\
\hline & Heteropterys grandiflora A.Juss. & LPQ 13680 & $\mathrm{x}$ & & trep & \\
\hline & Heteropterys trigoniifolia A.Juss. & DC 3034 & & $\mathrm{x}$ & trep & \\
\hline & Janusia schwannioides W.R.Anderson & LPQ 13673 & $\mathrm{x}$ & & trep & rl \\
\hline & Mcvaughia bahiana W.R.Anderson & GC 341, DC 958 & $\mathrm{x}$ & $\mathrm{x}$ & arb & end \\
\hline
\end{tabular}




\begin{tabular}{|c|c|c|c|c|c|c|}
\hline Família & Espécie & Voucher & $\mathbf{S}$ & C & Hábito & Obs. \\
\hline & Peixotoa sp. & DC 937 & $\mathrm{x}$ & & arb & \\
\hline & Ptilochaeta sp. & DC 2230 & $\mathrm{x}$ & & trep & \\
\hline & Stigmaphyllon auriculatum (Cav.) A.Juss. & GC 428 & & $\mathrm{x}$ & trep & \\
\hline & Stigmaphyllon paralias A.Juss. & DC 943 & $\mathrm{x}$ & & erv & \\
\hline & Stigmaphyllon salzmannii A.Juss. & GC 333 & & $\mathrm{x}$ & trep & \\
\hline & Tetrapterys paludosa A.Juss. & DC 963 & $\mathrm{x}$ & & trep & \\
\hline & Thryallis brachystachys Lindl. & DC 505 & & $\mathrm{x}$ & trep & \\
\hline & Thryallis longifolia Mart. & DC 48 & & $\mathrm{x}$ & trep & \\
\hline \multirow[t]{11}{*}{ Malvaceae } & Ceiba glaziovii (Kuntze) K.Schum. & DC 106 & & $\mathrm{x}$ & arv & \\
\hline & Helicteres velutina K.Schum. & GC 448 & $\mathrm{x}$ & & arb & \\
\hline & Helicteres vuarame Mart. & DC 894, GC 330 & $\mathrm{x}$ & $\mathrm{x}$ & arb & \\
\hline & Melochia betonicifolia A.St.-Hil. & GC 387 & & $\mathrm{x}$ & arb & \\
\hline & Pavonia sp. & & & & arb & \\
\hline & Pavonia blanchetiana Miq. & DC 955 & $\mathrm{x}$ & & arb & end \\
\hline & $\begin{array}{l}\text { Pseudobombax parvifolium Carv.-Sobr. \& } \\
\text { L.P.Queiroz }\end{array}$ & DC 502 & & $\mathrm{x}$ & arv & \\
\hline & Sida galheirensis Ulbr. & GC 405 & $\mathrm{x}$ & & arb & \\
\hline & Waltheria albicans Turcz. & DC 30 & $\mathrm{x}$ & & erv & \\
\hline & Waltheria brachypetala Turcz. & GC 374 & & $\mathrm{x}$ & erv & \\
\hline & Waltheria rotundifolia Schrank & DC 92 & & $\mathrm{x}$ & erv & \\
\hline \multirow[t]{3}{*}{ Melastomataceae } & Mouriri pusa Gardner & DC 193 & $\mathrm{x}$ & & arv & \\
\hline & Tibouchina caatingae J.G.Freitas & DC 533 & & $\mathrm{x}$ & arb & end \\
\hline & Tibouchina paulo-alvinii Vinha & DC 935 & $\mathrm{x}$ & & arb & \\
\hline \multirow[t]{2}{*}{ Meliaceae } & Trichilia hirta L. & GC 382 & & $\mathrm{x}$ & arv & \\
\hline & Cedrela odorata L. & DC 1329 & & $\mathrm{x}$ & arv & \\
\hline \multirow[t]{2}{*}{ Menispermaceae } & Cissampelos sp. & DC 497 & & $\mathrm{x}$ & trep & \\
\hline & Odontocarya duckei Barneby & DC 1303 & & $\mathrm{x}$ & trep & \\
\hline \multirow[t]{3}{*}{ Moraceae } & Brosimum gaudichaudii Trécul & DC 18, & $\mathrm{x}$ & & arb & \\
\hline & Ficus gomelleira Kunth & DC 212 & $\mathrm{x}$ & & arv & \\
\hline & Ficus mexiae Standl. & DC 173 & & $\mathrm{x}$ & arv & \\
\hline \multirow[t]{11}{*}{ Myrtaceae } & $\begin{array}{l}\text { Campomanesia eugenioides (Cambess.) } \\
\text { D.Legrand ex Landrum }\end{array}$ & GC 425 & $\mathrm{x}$ & & arv & \\
\hline & Eugenia candolleana DC. & GC 453 & $\mathrm{x}$ & & arv & \\
\hline & Eugenia dysenterica DC. & DC 2837 & $\mathrm{x}$ & & arv & \\
\hline & Eugenia ilhensis O.Berg. & DC 1316 & & $\mathrm{x}$ & arb & \\
\hline & Eugenia punicifolia (Kunth) DC. & DC 2720 & $\mathrm{x}$ & & arb & \\
\hline & Eugenia sp. & DC 925 & $\mathrm{x}$ & & arv & \\
\hline & Myrcia laruotteana Cambess. & DC 928 & $\mathrm{x}$ & & arb & \\
\hline & Myrcia polyantha DC. & GC 464 & $\mathrm{x}$ & & arb & \\
\hline & $\begin{array}{l}\text { Myrciaria floribunda (H.West ex Willd.) } \\
\text { O.Berg }\end{array}$ & AMC 3905 & $\mathrm{x}$ & & arb & \\
\hline & Psidium appendiculatum Kiaersk. & GC 336 & & $\mathrm{x}$ & arv & \\
\hline & Psidium schenckianum Kiaersk. & DC16, GC 453 & $\mathrm{x}$ & $\mathrm{x}$ & arb & \\
\hline
\end{tabular}




\begin{tabular}{|c|c|c|c|c|c|c|}
\hline Família & Espécie & Voucher & $\mathrm{S}$ & $\mathbf{C}$ & Hábito & Obs. \\
\hline \multirow[t]{2}{*}{ Nyctaginaceae } & Boerhavia difusa L. & LPQ 9032 & & $\mathrm{x}$ & erv & \\
\hline & Guapira tomentosa (Casar) Lundell & DC 2238 & $\mathrm{x}$ & & arb & \\
\hline \multirow[t]{2}{*}{ Ochnaceae } & Ouratea hexasperma (A.St.-Hil.) Baill. & DC 202 & $\mathrm{x}$ & & arb & \\
\hline & Ouratea parvifolia A.St. Hil. & AMC 3913 & $\mathrm{x}$ & & arb & \\
\hline Schoepfiaceae & Schoepfia brasiliensis A.DC. & GC 356 & & $\mathrm{x}$ & arv & \\
\hline \multirow[t]{2}{*}{ Onagraceae } & Ludwigia erecta (L.) H.Hara & GC 365 & $\mathrm{x}$ & & erv & \\
\hline & Ludwigia octovalvis (Jacq.) P.H.Raven & DC 76 & & $\mathrm{x}$ & erv & \\
\hline \multirow[t]{7}{*}{ Orchidaceae } & Alatiglossum barbatum (Lindl.) Baptista & DC 540 & & $\mathrm{x}$ & erv & \\
\hline & Campylocentrum crassirhizum Hoehne & DC 870 & & $\mathrm{x}$ & erv & \\
\hline & Catasetum purum Nees \& Sinning & DC 1325 & & $\mathrm{x}$ & erv & \\
\hline & Cohniella cebolleta (Jacq.) Christenson & DC 934 & $\mathrm{x}$ & & erv & \\
\hline & Pelexia sceptrum Schltr. & DC 3003 & & $\mathrm{x}$ & erv & \\
\hline & $\begin{array}{l}\text { Polystachya concreta (Jacq.) Garay \& } \\
\text { H.R.Sweet. }\end{array}$ & GC 396 & & $\mathrm{x}$ & erv & \\
\hline & Polystachya foliosa (Lindl.) Rchb.f. & DC 1328 & & $\mathrm{x}$ & erv & \\
\hline \multirow[t]{4}{*}{ Oxalidaceae } & Oxalis glaucescens Norlind. & DC 1203 & & $\mathrm{x}$ & erv & \\
\hline & Oxalis frutescens L. & DC 3027, GC 376 & $\mathrm{x}$ & $\mathrm{x}$ & erv & \\
\hline & Oxalis psoraleoides Kunth & LPQ 13666, GC 409 & $\mathrm{x}$ & $\mathrm{x}$ & erv & \\
\hline & Oxalis sp. & GC 424 & & $\mathrm{x}$ & erv & \\
\hline \multirow[t]{3}{*}{ Passifloraceae } & Passiflora edmundoi Sacco & GC 456 & $\mathrm{x}$ & & trep & \\
\hline & Passiflora foetida L. & DC 102 & & $\mathrm{x}$ & trep & \\
\hline & Passiflora luetzelburgii Harms & DC 191 & $\mathrm{x}$ & & trep & \\
\hline Phyllanthaceae & Phyllanthus flagelliformis Müll.Arg. & DC 953 & $\mathrm{x}$ & & erv & \\
\hline \multirow[t]{2}{*}{ Phytolaccaceae } & Microtea sp. & DC 970 & $\mathrm{x}$ & & trep & \\
\hline & Rivina humilis Lorenzi & LQ 9002 & & $\mathrm{x}$ & erv & \\
\hline Piperaceae & Peperomia blanda (Jacq.) Kunth & GC 388 & & $\mathrm{x}$ & erv & \\
\hline \multirow[t]{2}{*}{ Plantaginaceae } & Angelonia arguta Benth. & DC 60 & & $\mathrm{x}$ & erv & \\
\hline & Angelonia campestris Nees \& Mart. & DC 904 & $\mathrm{x}$ & & erv & \\
\hline Plumbaginaceae & Plumbago scandens L. & GC 867, DC 160 & $\mathrm{x}$ & $\mathrm{x}$ & arb & \\
\hline \multirow[t]{10}{*}{ Poaceae } & Paspalum arenarium Schrad. & DC 2709 & $\mathrm{x}$ & & erv & \\
\hline & Chloris barbata Sw. & AMC 3873 & & $\mathrm{x}$ & erv & \\
\hline & Eragrostis maypurensis (Kunth) Steud. & DC 2715, GC 413 & $\mathrm{x}$ & $\mathrm{x}$ & erv & \\
\hline & Setaria setosa (Sw.) P.Beauv. & GC 375 & & $\mathrm{x}$ & erv & \\
\hline & Lasiacis sp. & GC 332 & & $\mathrm{x}$ & erv & \\
\hline & Streptostachys sp. & DC 2705 & $\mathrm{x}$ & & erv & \\
\hline & Melinis repens (Willd.) Zizka & DC 538 & & $\mathrm{x}$ & erv & ex \\
\hline & Paspalum ligulare Nees. & AMC 3874 & & $\mathrm{x}$ & erv & \\
\hline & Panicum trichoides Sw. & DC 1341 & & $\mathrm{x}$ & erv & \\
\hline & Panicum venezuelae Hack. & DC 1338 & & $\mathrm{x}$ & erv & \\
\hline \multirow[t]{2}{*}{ Polygalaceae } & Acanthocladus albicans A.W.Benn. & DC 3014 & & $\mathrm{x}$ & arv & \\
\hline & $\begin{array}{l}\text { Asemeia mollis (Kunth) J.F.B.Pastore \& } \\
\text { J.R.Abbott }\end{array}$ & DC 93 & & $\mathrm{x}$ & erv & \\
\hline
\end{tabular}




\begin{tabular}{|c|c|c|c|c|c|c|}
\hline Família & Espécie & Voucher & $\mathrm{S}$ & C & Hábito & Obs. \\
\hline & $\begin{array}{l}\text { Asemeia parietaria (Chodat) J.F.B.Pastore \& } \\
\text { J.R.Abbott }\end{array}$ & GC 343 & & $\mathrm{x}$ & erv & \\
\hline & $\begin{array}{l}\text { Asemeia pseudohebeclada (Chodat) } \\
\text { J.F.B.Pastore \& J.R.Abbott }\end{array}$ & DC 1207 & $\mathrm{x}$ & & erv & \\
\hline & Caamembeca spectabilis (DC.) J.F.B.Pastore & DC 532 & & $\mathrm{x}$ & erv & \\
\hline & Polygala paniculata L. & DC 2718, 3023 & $\mathrm{x}$ & $\mathrm{x}$ & erv & \\
\hline & Polygala trichosperma Jacq. & DC 2716 & $\mathrm{x}$ & & erv & \\
\hline \multirow[t]{2}{*}{ Polygonaceae } & Coccoloba laevis Casar. & DC 93, 960 & & $\mathrm{x}$ & arb & \\
\hline & Ruprechtia laxiflora Meisn. & DC 956 & $\mathrm{X}$ & $\mathrm{x}$ & $\operatorname{arv}$ & \\
\hline \multirow[t]{2}{*}{ Polypodiaceae } & Pecluma plumula (Willd.) M.G.Price & DC 149 & & $\mathrm{x}$ & erv & \\
\hline & Pleopeltis sp. & DC 3023 & & $\mathrm{x}$ & erv & \\
\hline Pontederiaceae & Eichhornia paniculata (Spreng.) Solms & DC 75 & & $\mathrm{x}$ & erv & \\
\hline \multirow[t]{3}{*}{ Portulacaceae } & Portulaca mucronata Link & DC 913 & $\mathrm{x}$ & & erv & \\
\hline & Portulaca umbraticola Kunth & DC 484 & & $\mathrm{x}$ & erv & \\
\hline & Talinum paniculatum (Jacq.) Gaertn. & DC 161, GC 866 & $\mathrm{X}$ & $\mathrm{x}$ & erv & \\
\hline Proteaceae & Roupala montana Aubl. & DC 186 & $\mathrm{x}$ & & arb & \\
\hline \multirow[t]{2}{*}{ Rhamnaceae } & Ziziphus joazeiro Mart. & RPO 1429 & & $\mathrm{x}$ & $\operatorname{arv}$ & end \\
\hline & Ziziphus platyphylla Reissek & AMC 3853 & & $\mathrm{x}$ & arv & \\
\hline \multirow[t]{17}{*}{ Rubiaceae } & Chiococca alba (L.) Hitchc. & GC 338 & & $\mathrm{x}$ & arb & \\
\hline & Cordiera rigida (K.Schum.) Kuntze & DC 198,178 & $\mathrm{x}$ & & arv & \\
\hline & Cordiera sp. & GC 431 & & $\mathrm{x}$ & arv & \\
\hline & Coutarea hexandra (Jacq.) K.Schum. & GC 362, 445 & $\mathrm{x}$ & $\mathrm{x}$ & arv & \\
\hline & $\begin{array}{l}\text { Diodella gardneri (K.Schum.) Bacigalupo \& } \\
\text { E.L.Cabral }\end{array}$ & GC 404 & & $\mathrm{x}$ & arb & \\
\hline & $\begin{array}{l}\text { Diodella radula (Willd. ex Roem. \& Schult.) } \\
\text { Delprete }\end{array}$ & DC 210 & $\mathrm{x}$ & & erv & \\
\hline & Guettarda angelica Mart. ex Müll.Arg. & DC 893, 471 & $\mathrm{x}$ & $\mathrm{x}$ & arb & \\
\hline & Leptoscela ruellioides Hook.f. & GC 348 & & $\mathrm{x}$ & erv & \\
\hline & $\begin{array}{l}\text { Machaonia brasiliensis (Hoffmanns. ex } \\
\text { Humb.) Cham. \& Schltdl. }\end{array}$ & LPQ 9001 & & $\mathrm{x}$ & arb & \\
\hline & Manettia cordifolia Mart. & DC 548 & & $\mathrm{x}$ & trep & \\
\hline & $\begin{array}{l}\text { Mitracarpus frigidus (Willd.ex Roem.\&Schult.) } \\
\text { K.Schum. }\end{array}$ & GC 347 & & $\mathrm{x}$ & erv & \\
\hline & Mitracarpus baturitensis Sucre & GC 372 & & $\mathrm{x}$ & erv & $\mathrm{rl}$ \\
\hline & Mitracarpus salzmannianus DC. & DC 2710 & $\mathrm{x}$ & & erv & \\
\hline & Randia armata DC. & DC 504 & & $\mathrm{x}$ & arb & \\
\hline & $\begin{array}{l}\text { Staelia virgata (Link ex Roem.\& Schult.) } \\
\text { K.Schum. }\end{array}$ & AMC 3898 & $\mathrm{x}$ & & erv & \\
\hline & Staelia sp. & DC 2707 & $\mathrm{x}$ & & erv & \\
\hline & $\begin{array}{l}\text { Tocoyena formosa (Cham. \& Schltdl.) } \\
\text { K.Schum. }\end{array}$ & DC 473, AMC 3894 & $\mathrm{x}$ & $\mathrm{x}$ & arb & \\
\hline \multirow[t]{2}{*}{ Rutaceae } & Balfourodendron molle (Miq.) Pirani & DC 1209, 469 & $\mathrm{x}$ & $\mathrm{x}$ & arv & end \\
\hline & Zanthoxylum stelligerum Turcz. & DC 2722 & $\mathrm{x}$ & & arb & \\
\hline
\end{tabular}




\begin{tabular}{|c|c|c|c|c|c|c|}
\hline Família & Espécie & Voucher & $\mathbf{S}$ & $\mathbf{C}$ & Hábito & Obs. \\
\hline & Zanthoxylum petiolare A.St.-Hil. \& Tul. & DC 469 & & $\mathrm{x}$ & arv & \\
\hline Santalaceae & $\begin{array}{l}\text { Phoradendron liga (Gillies ex Hook. \& Arn.) } \\
\text { Eichler }\end{array}$ & DC 174 & $\mathrm{x}$ & & hem & \\
\hline \multirow[t]{6}{*}{ Sapindaceae } & Allophylus quercifolius (Mart.) Radlk. & LPQ 13679 & $\mathrm{x}$ & & arv & \\
\hline & Averrhoidium gardnerianum Baill. & DC 1182 & $\mathrm{x}$ & & arb & \\
\hline & Cardiospermum oliveirae Ferrucci & LPQ 183 & & $\mathrm{x}$ & trep & \\
\hline & Serjania glabrata Kunth & DC 125, 880 & $\mathrm{x}$ & & trep & \\
\hline & Urvillea andersonii Ferrucci & DC 73 & & $\mathrm{x}$ & trep & \\
\hline & Urvillea stipitata Radlk. & DC 126 & & $\mathrm{x}$ & trep & \\
\hline \multirow[t]{5}{*}{ Sapotaceae } & Chrysophyllum rufum Mart. & DC 208 & $\mathrm{x}$ & & arb & \\
\hline & Manilkara rufula (Miq.) H.J.Lam & DC 214 & $\mathrm{x}$ & & arv & \\
\hline & Pouteria gardneriana (A.DC.) Radlk. & DC 2057 & & $\mathrm{x}$ & arv & \\
\hline & Pouteria grandiflora (A.DC.) Baehni & DC 924 & $\mathrm{x}$ & & arv & \\
\hline & $\begin{array}{l}\text { Sideroxylon obtusifolium (Roem. \& Schult.) } \\
\text { T.D.Penn. }\end{array}$ & GC465, 414 & $\mathrm{x}$ & $\mathrm{x}$ & arv & \\
\hline Schizaeaceae & Anemia oblongifolia (Cav.) Sw. & GC 390 & $\mathrm{x}$ & & erv & \\
\hline Smilacaceae & Smilax campestris Griseb. & DC 201 & $\mathrm{x}$ & & trep & \\
\hline \multirow[t]{9}{*}{ Solanaceae } & Brunfelsia uniflora (Pohl) D.Don & DC 22 & $\mathrm{x}$ & & arb & \\
\hline & Schwenckia americana L. & GC 421 & & $\mathrm{x}$ & erv & \\
\hline & Solanum sp. 1 & GC 345 & & $\mathrm{x}$ & erv & \\
\hline & Solanum sp. 2 & GC 466 & $\mathrm{x}$ & & erv & \\
\hline & Solanum agrarium Sendtn. & DC 162 & & $\mathrm{x}$ & erv & \\
\hline & Solanum gardneri Sendtn. & DC 137 & & $\mathrm{x}$ & arb & \\
\hline & Solanum megalonyx Sendtn. & DC 1336 & & $\mathrm{x}$ & arb & \\
\hline & Solanum paniculatum L. & DC 127 & & $\mathrm{x}$ & arb & \\
\hline & Solanum stipulaceum Willd. ex Roem. & DC 21 & $\mathrm{x}$ & & arb & \\
\hline Trigoniaceae & Trigonia nivea Cambess. & DC 189 & $\mathrm{x}$ & & arb & \\
\hline \multirow[t]{6}{*}{ Turneraceae } & Piriqueta racemosa (Jacq.) Sw. & DC 483 & & $\mathrm{x}$ & erv & \\
\hline & Piriqueta sidifolia (Cambess.) Urb. & DC 1190 & $\mathrm{x}$ & & erv & \\
\hline & Turnera calyptrocarpa Urb. & DC 553 & & $\mathrm{x}$ & arb & \\
\hline & Turnera cearensis Urb. & LPQ 13672 & $\mathrm{x}$ & & erv & \\
\hline & Turnera chamaedrifolia Cambess. & GC 353, DC 487 & $\mathrm{x}$ & $\mathrm{x}$ & erv & \\
\hline & Turnera diffusa Willd. ex Schult. & $\begin{array}{l}\text { DC 1191, LPQ } \\
13678\end{array}$ & $\mathrm{x}$ & $\mathrm{x}$ & erv & \\
\hline Urticaceae & Laportea aestuans (L.) Chew & DC 1335 & & $\mathrm{x}$ & erv & \\
\hline Velloziaceae & Vellozia plicata Mart. & DC 539 & & $\mathrm{x}$ & erv & \\
\hline \multirow[t]{7}{*}{ Verbenaceae } & Lantana canescens Kunth & GC 452, LPQ 9010 & $\mathrm{x}$ & $\mathrm{x}$ & arb & \\
\hline & Lantana camara L. & DC 1321 & & $\mathrm{x}$ & arb & \\
\hline & Lippia sidioides Cham. & DC 914 & $\mathrm{x}$ & & arb & \\
\hline & Lippia microphylla Cham. & GC 401 & & $\mathrm{x}$ & arb & \\
\hline & Lippia origanoides Kunth & GC 364 & $\mathrm{x}$ & & arb & \\
\hline & Priva bahiensis A.DC. & GC 368 & & $\mathrm{x}$ & erv & \\
\hline & Stachytarpheta bicolor Hook.f. & DC 489 & & $\mathrm{x}$ & erv & \\
\hline
\end{tabular}




\begin{tabular}{lllllc}
\hline Família & Espécie & Voucher & S & C & Hábito Obs. \\
\hline Violaceae & Hybanthopsis bahiensis Paula-Souza & DC 938, 480 & x & x & trep \\
& Hybanthus verrucosus Paula-Souza & LPQ 9004 & & x & erv \\
Vitaceae & Cissus bahiensis Lombardi & DC 509 & & x & trep \\
& Cissus blanchetiana Planch. & DC 1334 & x & trep \\
& Cissus simsiana Schult. \& Schult.f. & DC 34, 56 & x & x & trep \\
& Cissus decidua Lombardi & DC 1302 & & x & trep \\
\hline
\end{tabular}

Na Depressão Sertaneja Meridional (DS), sobre solo derivado do embasamento cristalino, foram registradas 284 espécies, distribuídas em 213 gêneros e 62 famílias (Tab. 1). Leguminosae foi a família que apresentou a maior riqueza (58 spp.), seguida por Euphorbiaceae (20), Asteraceae (16), Rubiaceae (12), Malpighiaceae (8) e Apocynaceae (8). Senna foi o gênero mais rico em espécies (8 spp.), seguido por Chamaecrista, Cnidoscolus, Croton, Dioscorea e Jatropha, cada um com quatro espécies.

Na caatinga da ecorregião Raso da Catarina (RC), sobre o substrato arenoso derivado da bacia sedimentar, foram coletadas 226 espécies, classificadas em 169 gêneros e 61 famílias (Tab. 1). Leguminosae também foi a família mais rica (51 spp.), seguida por Euphorbiaceae (17), Malpighiaceae (14), Myrtaceae (10), Rubiaceae

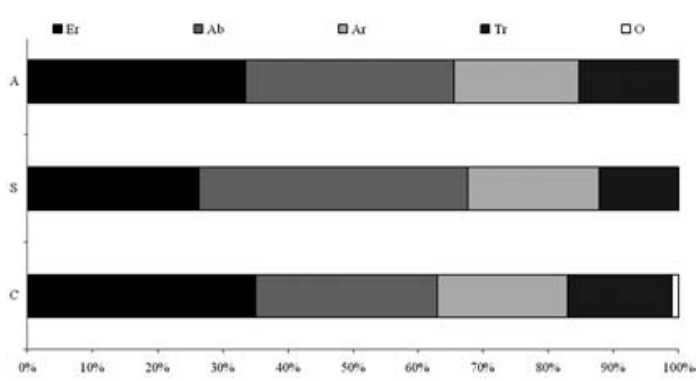

Figura 2 - Porcentagem de hábitos encontrados em caatinga sobre o embasamento cristalino derivado de rochas cristalinas pré-cambrianas $(\mathrm{S})$ e o substrato arenoso (A) originado de bacias sedimentares, município de Tucano, Bahia, Brasil. Hábitos: Árvore (Ar), Arbustos (Ab), Ervas (E), Trepadeiras (T) e outros (O).

Figure 2 - Percentage of habits found in caatinga on sandy sedimentary basin (S) and crystalline basement (C) and both areas (A) in the municipality of Tucano, northeastern of Bahia, Brazil. Habits: Tree (Ar), Shrub (Ab), Herb (E), Climbing (T) and Others $(\mathrm{O})$.
(8) e Apocynaceae (7). Croton, Chamaecrista e Mimosa foram os gêneros com maior riqueza, com seis espécies cada.

A proporção de espécies em comum nas duas ecorregiões foi 13\% (63/450), enquanto a de gêneros 43\% (91/213). Mais da metade dos gêneros (121/213) ocorrem exclusivamente na DS (57\%). A distribuição exclusiva de gêneros na RC foi de 37\% (79/213).

Foram identificadas 32 espécies endêmicas do Domínio da Caatinga (Tab. 1), das quais 15 com distribuição exclusiva na DS (por exemplo, Ditaxis desertorum), 11 na RC (por exemplo, Annona vepretorum, Calliandra aeschynomenoides) e seis em ambas as ecorregiões (por exemplo, Colicodendron yco).

Nove espécies ameaçadas de extinção foram registradas (Tab. 1), quatro exclusivamente associadas ao substrato arenoso (Ditassa arianeae Fontella \& E.A.Schwarz., Apocynaceae; Janusia schwannioides W.R.Anderson., Malpighiaceae; Mitracarpus baturitensis Sucre., Rubiaceae; Erythroxylum maracasense Plowman, Erythroxylaceae), três ao cristalino [Cyrtocarpa caatingae Mitchell \& Daly, Anacardiaceae; Blepharodon manicatum (Decne.) Fontella, Apocynaceae; Handroanthus selachidentatus (A.H.Gentry) S.O.Grose., Bignoniaceae] e duas em comum [Schinopsis brasiliensis Engl., Anacardiaceae; Chamaecrista belemii (H.S.Irwin \& Barneby) H.S.Irwin \& Barneby, Leguminosae].

A similaridade florística entre as duas ecorregiões foi de $25 \%$. Considerando apenas as ervas a similaridade foi de somente $7 \%$, enquanto trepadeiras 13\%, árvores 32\% e arbustos 33\%. O agrupamento encontrado com o NMDS aponta a separação das áreas conforme o substrato arenoso e do cristalino no Eixo $1(0,33)$, no Eixo $2(0,18)$ são evidenciadas aproximações de áreas de arenoso e cristalino, entre si, respectivamente (Fig. 3). 
Tabela 2 - Levantamentos florísticos em vegetação de caatinga sobre bacia sedimentar arenosa e embasamento cristalino. Table 2 - Floristic surveys on caatinga vegetation on sandy soil and crystalline basement.

\begin{tabular}{|c|c|c|c|c|c|}
\hline $\begin{array}{l}\mathrm{N}^{\circ} \text { de } \\
\text { espécies }\end{array}$ & $\begin{array}{l}\mathrm{N}^{\circ} \text { Espécies de } \\
\text { Leguminosae }\end{array}$ & Geologia & Fitofisionomia & Localidade & Referência \\
\hline 136 & 35 & Sedimentar & Savana/Caatinga/Carrasco & São José do Piauí, PI & Mendes \& Castro (2010) \\
\hline 210 & 64 & Sedimentar/Cristalino & Caatinga arbórea à arbustiva & São Raimundo Nonato, PI & Lemos (2004) \\
\hline 192 & 29 & Sedimentar & Vegetação perenifólia arbustiva & Buíque, PE & Gomes et al. (2006) \\
\hline 211 & 18 & Cristalino & Caatinga com áreas rochosas & Agrestina, PE & Gomes \& Alves (2010) \\
\hline 144 & 14 & Cristalino & Caatinga com áreas rochosas & Venturosa, PE & Gomes et al. (2011) \\
\hline 96 & 17 & Cristalino & $\begin{array}{l}\text { Vegetação caducifólia } \\
\text { espinhosa arbórea }\end{array}$ & Caruaru, PE & Alcoforado-Filho et al. (2003) \\
\hline 174 & 29 & Cristalino & Floresta hiperxerófila densa & $\begin{array}{l}\text { Canindé do São Francisco/ } \\
\text { Poço Redondo, SE }\end{array}$ & Silva et al. (2013) \\
\hline 133 & 22 & Cristalino & Caatinga arbórea/Savana & Não Me Deixes, CE & Costa et al. (2007) \\
\hline 101 & 10 & Cristalino & Caatinga & Floresta e Betânia, PE & Costa et al. (2009) \\
\hline 184 & 42 & Sedimentar & Carrasco & Novo Oriente, CE & Araújo et al. (1998) \\
\hline 86 & 24 & Sedimentar & Caatinga arbustiva & Barra, BA & Rocha et al. (2004) \\
\hline 186 & 32 & Cristalino & Caatinga arbustiva densa & Iguatu, CE & Lima (2012) \\
\hline 148 & 35 & Sedimentar & Caatinga arbustiva densa & Iguatu, CE & Lima (2012) \\
\hline 104 & 20 & Sedimentar & Floresta estacional decídua & Crateús, CE & Lima et al. (2009) \\
\hline 284 & 58 & Cristalino & Caatinga arbórea à arbustiva & Tucano, BA & Presente estudo \\
\hline 226 & 51 & Sedimentar & Caatinga arbustiva & Tucano, BA & Presente estudo \\
\hline 450 & 97 & Cristalino/Sedimentar & Caatinga arbórea à arbustiva & Tucano, BA & Presente estudo \\
\hline
\end{tabular}

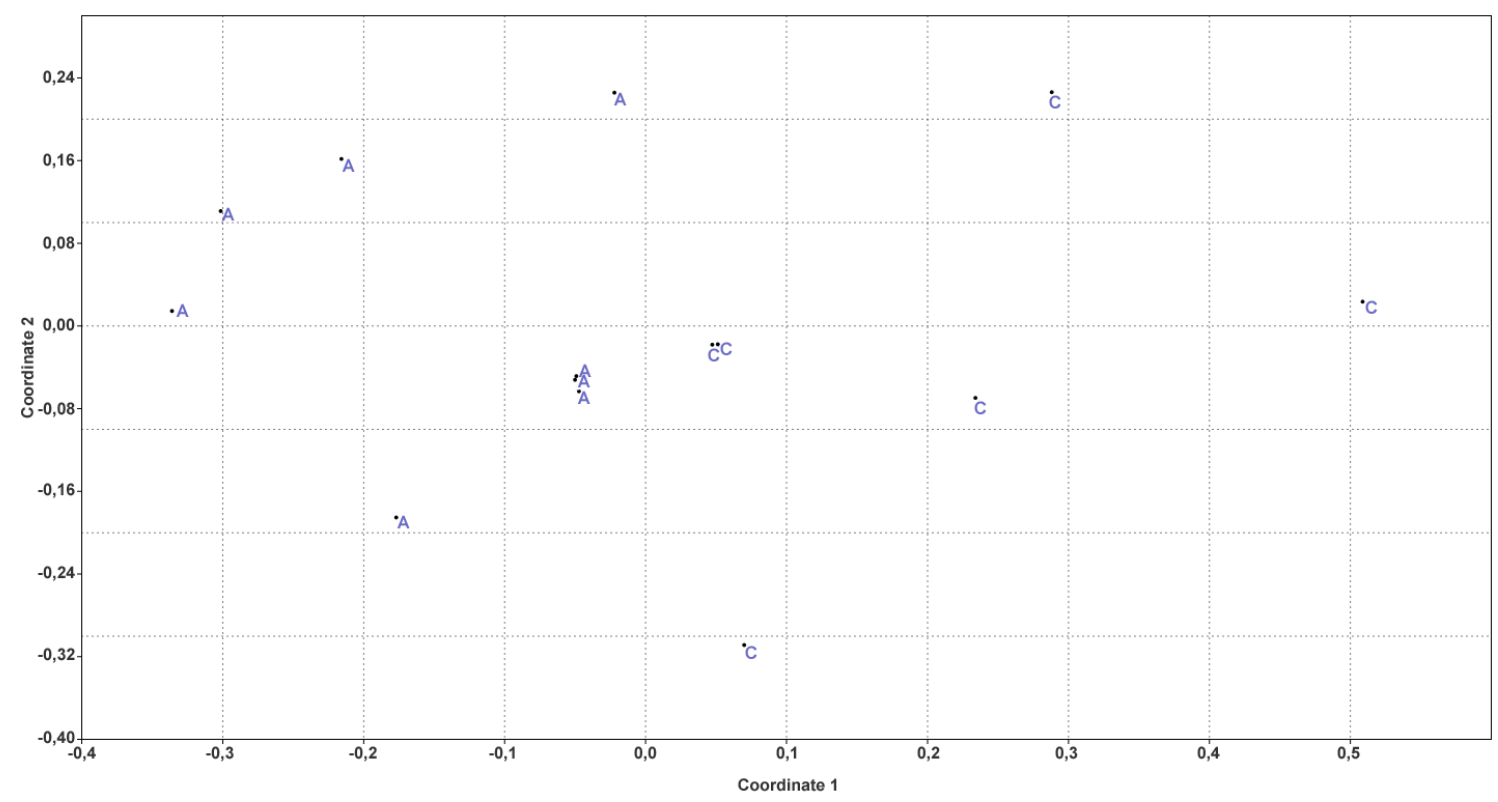

Figura 3 - Ordenação baseada em presence e ausência de espécies ocorrendo em 14 fragmentos de caatinga sobre o embasamento cristalino derivado de rochas cristalinas pré-cambrianas (C) e o substrato arenoso (S) originado de bacias sedimentares, e ambas as áreas (A), município de Tucano, Bahia, Brasil.

Figure 3 - NMDs ordination plot based in presence/absence of species occurring in 14 locations of caatinga on sandy sedimentary basin (A) and crystalline basement (C) in the municipality of Tucano, northeastern Bahia, Brazil. 


\section{Discussão}

Ainda existe grande deficiência de conhecimento florístico da vegetação de caatinga quando comparado aos demais biomas, como as florestas úmidas (Barbosa et al. 2005). Provavelmente, isso se deve em grande parte à insuficiência de coletas (Tabarelli \& Vicente 2004), que devido também à sazonalidade da vegetação, necessita ser constantemente inventariada. A lacuna de conhecimento especificamente sobre as caatingas da Bahia, quando comparada aos demais estados do semiárido, incide no fato de existirem poucos trabalhos publicados de inventários florísticos e fitossociológicos (Moro et al. 2014). Entretanto, investimentos em coletas sistemáticas têm de fato acontecido nos últimos dez anos no Domínio Fitogeográfico das Caatingas (Moro et al. 2015), de modo que a elevada riqueza das caatingas da Bahia pode ser atestada pela recorrente descoberta de espécies novas em diferentes famílias (Cardoso \& Queiroz 2008; Cardoso et al. 2008; Queiroz \& Cardoso 2008; Carvalho-Sobrinho \& Queiroz 2008, 2010; Silva \& Rapini 2009; Côrtes \& Rapini 2011; Queiroz \& Lavin 2011; Roque \& Funk 2011; Carvalho-Sobrinho et al. 2012, 2014; Machado \& Queiroz 2012; Freitas et al. 2013; Carvalho \& Queiroz 2014; Almeida \& Amorim 2014), incluindo os novos gêneros endêmicos Tabaroa L.P.Queiroz, G.P.Lewis \& M.F.Wojc. (LeguminosaePapilionoideae) (Queiroz et al. 2010) e Keraunea Cheek \& Simão-Bianchini (Convolvulaceae) (Cheek \& Simão-Bianchini 2013), e até mesmo o novo registro da família Hydnoraceae no estado, através da descoberta da nova espécie Prosopanche caatingicola R.F.Machado \& L.P.Queiroz (Machado \& Queiroz 2012).

Toda a flora inventariada nas duas ecorregiões de Tucano representa cerca de 30\% do número de espécies apontado nas caatingas (Queiroz et al. 2006), fruto de investimento intensivo em coleta, onde foi possível explorar mais de uma localidade. Estes números representam uma riqueza de espécies muito superior aos levantamentos florísticos em outras áreas de caatinga do Nordeste, tanto os realizados em substrato derivado do embasamento cristalino (Costa et al. 2007; Roque et al. 2009; Lima 2012; Silva et al. 2013) quanto àqueles em substrato arenoso derivado da bacia sedimentar (Araújo et al. 1998; Rocha et al. 2004; Gomes et al. 2006; Mendes \& Castro 2010; Lima 2012). A riqueza média observada em estudos florísticos de vegetação de caatinga é de 106 espécies (variando de 21 a 250 espécies; Moro et al. 2015) (Tab. 2). Apenas tomando como comparação as Leguminosae, frequentemente a família mais representativa em qualquer inventário na caatinga, a diversidade de 97 espécies encontrada nas caatingas de Tucano é também maior do que em muitos levantamentos já realizados no nordeste semiárido (Tab. 2). Além disso, as novas espécies Senna bracteosa (Cardoso \& Queiroz 2008), Pseudobombax parvifolium (Carvalho-Sobrinho \& Queiroz 2010) e Tibouchina caatingae (Freitas et al. 2013), descritas recentemente a partir de materiais-tipo coletados em Tucano, atestam o desconhecimento da flora das caatingas, contribuindo para o reconhecimento da elevada diversidade florística nas caatingas de Tucano.

A maior riqueza e elevado endemismo em espécies de Leguminosae nas duas ecorregiões reforçam a sua condição de principal família nas FTSS (Gentry 1995; Schrire et al. 2005; Queiroz et al. 2006; Cardoso \& Queiroz 2011). As leguminosas frequentemente aparecem em destaque em inventários de caatingas, seja em formações sobre embasamento cristalino (Costa et al. 2009; Lima 2012; Machado et al. 2012), substrato arenoso derivado da bacia sedimentar (Rocha et al. 2004), ou em áreas com predominância de afloramento rochoso (Araújo et al. 2008; Gomes \& Alves 2010), como também em levantamentos restritos ao componente arbustivoarbóreo (Lemos \& Rodal 2002) e nos que incluem os demais hábitos (Alcoforado-Filho et al. 2003; Costa et al. 2007; Costa et al. 2009; Machado et al. 2012). Neste trabalho, as coletas resultaram em um acréscimo de 25 espécies e 21 gêneros à listagem de Leguminosae nas caatingas de Tucano (Cardoso \& Queiroz 2007).

A riqueza de Euphorbiaceae é também relevante na caatinga (Queiroz et al. 2006; Carneiro-Torres 2009; Moro et al. 2014), porém sua maior expressividade acontece quando considerado o estrato arbustivo-arbóreo, tanto em áreas sobre substrato arenoso derivado da bacia sedimentar (Araújo et al. 1998; Rocha et al. 2004; Gomes et al. 2006), quanto àquelas sobre embasamento cristalino (Costa et al. 2009; Lima 2012; Machado et al. 2012). Entretanto, em outros núcleos de florestas secas, a diversidade de Euphorbiaceae não é tão expressiva como na caatinga (Gentry 1995). Enquanto Euphorbiaceae é frequentemente referida como a segunda família mais diversa em inventários florísticos na Caatinga, geralmente 
ela é superada por Rubiaceae em florestas secas da América Central (Gillespie et al. 2000) e Ásia (Krishnamurthy et al. 2010).

A maioria das espécies de Malpighiaceae ocorreu na caatinga da RC. Esta família tem sido de fato apontada como importante componente da flora da caatinga (Queiroz et al. 2006, OliveiraFilho et al. 2013). Os dados do presente inventário, quando associados a estudos em outras caatingas sobre substrato arenoso, sugerem uma predileção dessa família por áreas arenosas. Nos levantamentos florísticos em caatinga sobre substrato arenoso (incluindo a fitofisionomia de carrasco), verifica-se a ocorrência frequente de Byrsonima gardneriana (Araújo et al. 1998; Lemos \& Rodal 2002; Cestaro \& Soares 2004; Rocha et al. 2004; Gomes et al. 2006).

Myrtaceae também apresentou elevada riqueza no presente estudo, apesar de não ser uma família bem representada na caatinga (Queiroz et al. 2006) e demais florestas secas neotropicais (Gentry 1995; Lucas et al. 2012). No entanto, as espécies de Myrtaceae têm sido reportadas na caatinga preferencialmente em áreas sobre substrato arenoso (Lemos \& Rodal 2002; Gomes et al. 2006). Neste estudo, não pudemos detectar espécies que realmente possam ser utilizadas como indicadoras exclusivamente de caatinga arenosa. Por tratar-se de uma família taxonomicamente complexa, não podemos descartar a possibilidade de que o pouco conhecimento sobre as espécies de Myrtaceae na caatinga pode ser fruto de problemas de identificação em nível de espécie na maioria das listas florísticas disponíveis (Moro et al. 2014).

Em contraste com Myrtaceae, Asteraceae apresentou maior riqueza nas caatingas sobre substrato derivado do embasamento cristalino, mas igualmente não foram evidenciadas espécies com maior predileção por estas áreas. No caso das Asteraceae, a falta de espécies indicadoras das diferentes fitofisionomias de caatinga provavelmente se deve ao fato da família ser muito mais representada por espécies de ampla distribuição em todo o domínio. No entanto, não podemos deixar de destacar que a escassez de informações sobre o estrato herbáceo em inventários florísticos de caatinga prejudica comparações e análises mais abrangentes sobre endemismos, diversidade e distribuição em famílias notavelmente marcadas por espécies herbáceas, a exemplo de Asteraceae e Rubiaceae (Queiroz et al. 2006; Silva et al. 2013; Moro et al. 2014). Acreditase que trabalhos florísticos focados também em outros hábitos, e não apenas no componente arbustivo-arbóreo como geralmente tem sido feito (Rodal \& Nascimento 2006), permitirá uma melhor caracterização e delimitação biogeográfica das diferentes fisionomias de Caatinga, e como estas se relacionam com os demais núcleos de FTSS. Embora as ervas sejam menos conhecidas na caatinga, devido principalmente à dificuldade de coletas ocasionada pela efemeridade da maioria das espécies (Reis et al. 2006), sínteses ou comparações florísticas que tentam incorporar informações sobre o estrato herbáceo poderá beneficiar a compreensão da fitogeografia da caatinga, uma vez que os números recentes mostram que as ervas são de fato relativamente bem representadas (Costa et al. 2007). Neste estudo, revelou-se a importância do estrato herbáceo na composição florística das duas ecorregiões de Tucano, ao mostrarmos que este estrato ocorre em proporções similares ou até mesmo superiores em relação ao componente arbustivo-arbóreo. A riqueza total de ervas nas caatingas de Tucano corrobora com a elevada diversidade de espécies herbáceas na caatinga, com riqueza superior até mesmo em comparação com outros tipos de vegetação (Santos et al. 2013; Silva et al. 2013). Silva et al. (2013) registraram elevada similaridade de ervas entre caatingas sobre embasamento cristalino (DS) e solo arenoso (RC), o que difere consideravelmente do presente estudo, no qual a similaridade entre as ervas foi a menor entre todos os hábitos considerados.

Os padrões de diversidade de espécies e hábitos predominantes nas diferentes famílias, discutidos acima em escala local com as ecorregiões DS e RC em Tucano, confirmam a hipótese de que os tipos de substrato geologicamente diferentes no Domínio da Caatinga permitem a divisão de duas unidades florísticas formadas por biotas historicamente distintas: "caatinga de areia" em superfícies sedimentares e "caatinga do cristalino representada pela vegetação em superfícies expostas do embasamento cristalino" (Araújo et al. 2005; Queiroz 2006; Cardoso \& Queiroz 2007; Santos et al. 2012). A distribuição e endemismo em nível genérico têm sido bastante informativos na classificação e delimitação fitogeográfica dos principais núcleos de florestas secas (Prado 2000; López et al. 2006) e destas com florestas úmidas (Rodal et al. 2008).

A similaridade de $25 \%$, ou seja, alta betadiversidade entre as floras das ecorregiões DS e RC, acrescida da distinção evidenciada pela análise de 
agrupamento, com maior adensamento em áreas com maior esforço de coleta, atestam que nestas ecorregiões, embora geograficamente próximas, evoluíram biotas modeladas por diferentes fatores históricos (Queiroz 2006). Análises espacial e multivariada com dados de distribuição de 1332 espécies arbóreas em 187 áreas de florestas secas (Santos et al. 2012) também sugerem que a “caatinga arenosa” deveria ser reconhecida como uma unidade florística distinta. A existência de biotas distintas na caatinga nos leva a questionar, portanto, a relação histórica de toda a caatinga com os demais núcleos de FTSS. A caatinga tem sido rotineiramente discutida na literatura, através de padrões de distribuição de espécies arbóreas, como uma unidade vegetacional com a mesma história biogeográfica das FTSS (Sarmiento 1975; Prado \& Gibbs 1993; Prado 2000; Pennington et al. 2000; Oliveira-Filho et al. 2006). Muito provavelmente a biota da caatinga mais relacionada com as FTSS neotropicais é aquela pertencente às áreas sobre solos derivados do embasamento cristalino, que na região de Tucano corresponde à DS. Esta sugestão havia sido detalhadamente investigada com base em síndromes de dispersão, padrões fenológicos e de diversidade e endemismo na vegetação das dunas do vale médio do Rio São Francisco (Rocha et al. 2004), bem como a partir dos padrões biogeográficos de Leguminosae (Queiroz 2006; Cardoso \& Queiroz 2007). No presente estudo, corrobora-se em escala local também a partir da distribuição de outras famílias, que não apenas as leguminosas. As espécies características das FTSS que ocorrem na região de Tucano e que são sempre associadas à DS, são as Leguminosae Amburana cearensis, Anadenanthera colubrina, Geoffroea spinosa, Mimosa tenuiflora e Parkinsonia aculeata, além de Handroanthus impetiginosus (Bignoniaceae), Trichilia hirta (Meliaceae), Coutarea hexandra (Rubiaceae), Pouteria gardneriana, Sideroxylon obtusifolium (Sapotaceae), Cordia trichotoma, Varronia globosa, $V$. leucocephala (Boraginaceae) e Ziziphus joazeiro (Rhamnaceae). Este número é muito maior do que as espécies de ampla distribuição nas FTSS e que também se distribuem na caatinga arenosa da RC, que contam somente com Astronium fraxinifolium (Anacardiaceae), Brosimum gaudichaudii (Moraceae), Ruprechtia laxiflora (Polygonaceae) e as leguminosas Pithecellobium diversifolium e Hymenaea martiana.

\section{Considerações Finais}

A importância do substrato na determinação da composição florística em caatinga foi aqui demonstrada em escala local, assim como tem sido apontada em escalas mais amplas (Rodal 1992; Araújo et al., 2005, Gomes et al. 2006, Queiroz 2006; Cardoso \& Queiroz 2007; Santos et al. 2012). Em conjunto, estes resultados reforçam o reconhecimento da heterogeneidade florística na caatinga associada aos substratos geologicamente distintos. Diversos táxons com distribuição restrita, diferenças nos hábitos predominantes e baixa similaridade têm sido padrões detectados na composição de Leguminosae em diferentes fitofisionomias de caatinga do Nordeste brasileiro (Cardoso \& Queiroz 2007) e, no presente estudo, ratificados principalmente pelos padrões de diversidade e distribuição de Euphorbiaceae, Malpighiaceae e Myrtaceae.

A necessidade de conservação da Caatinga é notória (Castelletti et al. 2003; Leal et al. 2005; Melo et al. 2014), sendo Tucano área prioritária para a conservação, já que possui cerca de 30\% das espécies listadas para a vegetação de caatinga (Queiroz et al. 2006) e compartilha remanescentes de vegetação nativa sobre substratos geologicamente distintos. A alta diversidade florística desse município deve-se em grande parte pela distribuição diferenciada das espécies entre os substratos, com distinção nos hábitos predominantes e alta taxa de endemismo.

\section{Agradecimentos}

Agradecemos imensamente o apoio de Timóteo, Jeová Moura e João Moura em coletas na Quixaba do Mandacaru, e Camila Vitório, Douglas Moreira, Jonatas Amorim, Alexandre Miranda, Ademário Bastos e Márcio Harrison Ferreira em ajuda de campo em diversas outras localidades. GMC agradece à CAPES, a bolsa de Doutorado; aos especialistas que identificaram e/ou confirmaram a determinação de espécies: Alessandro Rapini (Apocynaceae), Ana Luiza Côrtes (Acanthaceae), Cecília Azevedo (Orchidaceae), Daniela Carneiro-Torres (Euphorbiaceae), Efigênia de Melo (Polygonaceae), Jefferson de CarvalhoSobrinho (Malvaceae-Bombacoideae), Juliana Freitas (Melastomataceae), Karoline Coutinho (Myrtaceae), Lamarck Rocha (Turneraceae), Lidyanne Aona (Commelinaceae), Liziane Vasconcelos (Convolvulaceae), Márcio Martins 
(Euphorbiaceae), Marla Ibrahim (Myrtaceae), Matheus Nogueira (Bromeliaceae), Nelma Sousa (Onagraceae), Rafael Almeida (Malpighiaceae), Reyjane Patrícia Oliveira (Poaceae); aos funcionários do HUEFS e HURB por montarem e organizarem coleções de plantas das caatingas de Tucano.

\section{Referências}

Ab’Saber, A.N. 1974. O domínio morfoclimático semiárido das caatingas brasileiras. Geomorfologia 43: 1-39.

Albuquerque, U.P.; Araújo, E.L.; El-Deir, A.C.A.; Lima, A.L.A.; Souto, A.; Bezerra, B.M.; Ferraz, E.M.N.; Freire, E.M.X.; Sampaio, E.V.S.B; Las-Casas, F.M.G.; Moura, G.J.B.; Pereira, G.A.; Melo, J.G.; Ramos, M.A.; Rodal, M.J.N.; Schiel, N.; LyraNeves, R.M.; Alves, R.R.N.; Azevedo-Júnior, S.M.; Telino-Júnior, W.R. \& Severi, W. 2012. Caatinga revisited: Ecology and conservation of an important seasonal dry forest. The Scientific World Journal 205182: 1-18.

Alcoforado-Filho, F.G.; Sampaio, E.V.S.B. \& Rodal, M.J.N. 2003. Florística e fitossociologia de um remanescente de vegetação caducifólia espinhosa arbórea em Caruaru, Pernambucano. Acta Botanica Brasilica 17: 287-303.

Almeida, R.F. \& Amorim, A.M. 2014. Stigmaphyllon caatingicola (Malpighiaceae), a new species from Seasonally Dry Tropical Forests in Brazil. Phytotaxa 174: 82-88.

Andrade-Lima, D. 1981. The caatingas dominium. Revista Brasileira de Botânica 4: 149-163.

APG III. 2009. An update of the Angiosperm Phylogeny Group classification for the orders and families of flowering plants: APG III. Botanical Journal of the Linnean Society 161: 105-121.

Araújo, F.S.; Costa, R.C.; Lima, J.R.; Vasconcelos, S.F.; Girão, L.C.; Souza Sobrinho, M.; Bruno, M.M.A.; Souza, S.S.G.; Nunes, E.P.; Figueiredo, M.A.; LimaVerde, L.W. \& Loiola, M. I.B. 2011. Floristics and life-forms along a topographic gradient, centralwestern Ceará, Brazil. Rodriguésia 62: 341-366.

Araújo, F.S.; Oliveira, R.F. \& Lima-Verde, L.W. 2008. Composição, espectro biológico e síndromes de dispersão na vegetação de um inselbergue no domínio da Caatinga, Ceará. Rodriguésia 59: 659-671.

Araújo, F.S.; Rodal, M.J.N; Barbosa, M.R.V \& Martins, F.R. 2005. Repartição da flora lenhosa no domínio da Caatinga. In: Araújo, F.S.; Rodal, M.J.N. \& Barbosa, M.R.V. (orgs). Análise das variações da biodiversidade do bioma caatinga: suporte a estratégias regionais de conservação. Ministério do Meio Ambiente; Brasília. Pp. 15-33.
Araújo, F.S.; Sampaio. E.V.S.B.; Figueiredo, M.A.; Rodal, M.J.N. \& Fernandes, A.G. 1998. Composição florística da vegetação de carrasco, Novo Oriente, CE. Revista Brasileira de Botânica 21: 105-116.

Barbosa, M.R.V.; Castro, R.; Araújo, F.S. \& Rodal, M.J.N. 2005. Estratégias para conservação da biodiversidade e prioridades para a pesquisa científica no bioma Caatinga. In: Araújo, F.S. Rodal, M.J.N. \& Barbosa, M.R.V. (eds.). Análise das variações da biodiversidade do Bioma Caatinga: Suporte a estratégias regionais. Ministério do Meio Ambiente; Brasília. Pp. 417-431.

Cardoso, D.B.O.S. \& Queiroz, L.P. 2007. Diversidade de Leguminosae nas caatingas de Tucano, BA: Implicações para a fitogeografia do semi-árido do Nordeste do Brasil. Rodriguésia 58: 379-371.

Cardoso, D.B.O.S. \& Queiroz, L.P. 2008. A new species of Senna (Leguminosae, Caesalpinioideae) from Eastern Brazil. Novon 18: 140-143.

Cardoso, D.B.O.S. \& Queiroz, L.P. 2011. Caatinga no contexto de uma metacomunidade: evidências da biogeografia, padrões filogenéticos e abundância de espécies em Leguminosas. In: Carvalho, C.J.B.; Almeida, E.A.B. (eds.). Biogeografia da América do Sul: padrões e processos. Roca, São Paulo. Pp. 241-260.

Cardoso, D.B.O.S.; Queiroz, L.P. \& Lima, H.C. 2008. Three new species of Luetzelburgia (Leguminosae, Papilionoideae) from the Caatinga of Bahia, Brazil and an identification key to all species of the genus. Kew Bulletin 63: 289-300.

Carneiro-Torres, D.S. 2009. Diversidade de Croton L. (Euphorbiaceae) no Bioma Caatinga. Tese de Doutorado. Universidade Estadual de Feira de Santana, Feira de Santana. 295p.

Carvalho, M.L.S. \& Queiroz, L.P. 2014. Philcoxia tuberosa (Plantaginaceae), a new species from Bahia, Brazil. Neodiversity 7: 14-20.

Carvalho-Sobrinho, J.G. \& Queiroz, L.P. 2008. Ceiba rubriflora (Malvaceae: Bombacoideae), a new species from Bahia, Brazil. Kew Bulletin 63: 649-653.

Carvalho-Sobrinho, J.G. \& Queiroz, L.P. 2010. Three new species of Pseudobombax (Malvaceae, Bombacoideae) from Brazil. Novon 20: 13-20.

Carvalho-Sobrinho, J.G.; Alverson, W.S.; Mota, A.C.; Machado, M.C. \& Baum, D.A. 2014. A new deciduous species of Pachira (Malvaceae: Bombacoideae) from a Seasonally Dry Tropical Forest in Northeastern Brazil. Systematic Botany 39: 260-267.

Carvalho-Sobrinho, J.G.; Machado, M.C. \& Queiroz, L.P. 2012. Spirotheca elegans (Malvaceae: Bombacoideae), a new species from Bahia, Brazil. Systematic Botany 37: 978-982.

Castelletti, C.H.M.; Santos A.M.M.; Tabarelli, M. \& Silva, J.M.C. 2003. Quanto ainda resta da Caatinga? 
Uma estimativa preliminar. In: Leal, I.R.; Tabarelli, M. \& Silva, J.M.C. (eds.). Ecologia e conservação da Caatinga. Editora Universitária, Universidade Federal de Pernambuco, Recife. Pp. 719-734.

Cestaro, L.A. \& Soares, J.J. 2004. Variações florística e estrutural e relações fitogeográficas de um fragmento de floresta decídua no Rio Grande do Norte, Brasil. Acta Botanica Brasilica 18: 203-218.

Cheek, M. \& Simão-Bianchini, R. 2013. Keraunea gen. nov. (Convolvulaceae) from Brazil. Nordic Journal of Botany 31: 453-457.

Côrtes, A.L.A. \& Rapini, A. 2011. Four new species of Justicia (Acanthaceae) from the Caatinga biome of Bahia, Brazil. Kew Bulletin 66: 453-461.

Costa, K.C.; Lima, A.L.A.; Fernandes, C.H.M.; Silva, M.C.N.A.; Silva, A.C.B.L. \& Rodal, M.J.N. 2009. Flora vascular e formas de vida em um hectare de caatinga no Nordeste brasileiro. Revista Brasileira de Ciências Agrárias 4: 48-54.

Costa, R.C.; Araújo, F.S. \& Lima-Verde, L.W. 2007. Flora and life-form spectrum in an area of deciduous thorn woodland (caatinga) in northeastern, Brazil. Journal of Arid Environments 68: 237-247.

Forzza, R.C.; Baumgratz, J.F.A.; Bicudo, C.E.M.; Carvalho-Jr., A.A.; Costa, A.; Costa, D.P.; Hopkins, M.; Leitman, P.M.; Lohmann, L.G.; Maia, L.C.; Martinelli, G.; Menezes, M.; Morim, M.P.; Coelho, M.A.N.; Peixoto, A.L.; Pirani, J.R.; Prado, J., Queiroz, L.P., Souza, V.C., Stehmann, J.R., Sylvestre, L.S., Walter, B.M.T. \& Zappi, D. (eds.). 2010. Catálogo de plantas e fungos do Brasil. Vol. 2. Andrea Jakobsson Estúdio/Instituto de Pesquisas Jardim Botânico do Rio de Janeiro, Rio de Janeiro. 831p.

Freitas, J.G.; Santos, A.K.A.; Guimarães, P.J.F. \& Oliveira, R.P. 2013. A new and unusual species of Tibouchina (Melastomataceae) occurring in caatinga vegetation in Bahia, Brazil. Systematic Botany 38: 418-423.

Funk, V.A. \& Roque, N. 2011. The monotypic Andean genus Fulcaldea (Compositae, Barnadesioideae) gains a new species from northeastern Brazil. Taxon 60: 1095-1103.

Gentry, A.H. 1995. Diversity and floristic composition of neotropical dry forests. In: Bullock, S.H.; Mooney, H.A. \& Medina, E. (eds.). Seasonally dry tropical forests. Cambridge University Press, Cambridge. Pp. 146-194.

Gillespie, T.W.; Grijalva, A. \& Farris, C.N. 2000. Diversity, composition and structure of tropical dry forests in Central America. Plant Ecology 147: 37-47.

Giulietti, A.M.; Harley, R.M.; Queiroz, L.P.; Barbosa M.R.V.; Bocage-Neta A.L. \& Figueiredo, M.A. 2002. Espécies endêmicas da caatinga. In: Sampaio, E.V.B.; Giulietti, A.M.; Virgínio, J. \& GamarraRojas, C. (eds.). Vegetação e flora da caatinga. Associação Plantas do Nordeste - APNE/CNIP, Recife. Pp. 103-118.
Gomes, A.P.S.; Rodal, M.J.N. \& Melo, A.L. 2006. Florística e fitogeografia da vegetação arbustiva subcaducifólia da Chapada de São José, Buíque, PE, Brasil. Acta Botanica Brasilica 20: 37-48.

Gomes, P. \& Alves, M. 2010. Floristic diversity of two crystalline rocky outcrops in the Brazilian northeast semi-arid region. Revista Brasileira de Botanica 33: 661-676.

Gomes, P.; Costa, K.C.C.; Rodal, M.J.N. \& Alves, M. Checklist of Angiosperms from the Pedra Furada Municipal Park, northeastern Brazil. Check List 7: 173-181.

Hammer, Ø., Harper, D.A.T., Ryan, P.D. 2001. PAST: Paleontological statistics software package for education and data analysis. Palaeontologia Electronica 4: 9.

Krishnamurthy, Y.L.; Prakasha, H.M.; Nanda, A.; Krishnappa, M.; Dattaraja, H.S. \& Suresh, H.S.. 2010. Vegetation structure and floristic composition of a tropical dry deciduous forest in Bhadra Wildlife Sanctuary, Karnataka, India. Tropical Ecology 51: 235-246.

Leal, I.R.; Silva, J.M.C.; Tabarelli, M. \& Lacher-Jr., T. 2005. Mudando o curso da conservação da biodiversidade na Caatinga do nordeste do Brasil. Megadiversidade 1: 139-146.

Lemos, J.R. \& Rodal, M.J.N. 2002. Fitossociologia do componente lenhoso de um trecho da vegetação de caatinga no Parque Nacional Serra da Capivara, Piauí, Brasil. Acta Botanica Brasilica 16: 23-42.

Lemos, J.R. 2004. Composição florística do Parque Nacional Serra da Capivara, Piauí, Brasil. Rodriguésia 55: 55-66.

Lewis, G.P.; Schrire, B.D.; Mackinder, B.A. \& Lock, M. 2005. Legumes of the world. Royal Botanic Gardens, Kew. 577p.

Lima, B.G. 2012. Caatinga: Espécies lenhosas e herbáceas. EDUFERSA, Mossoró. 316p.

Linares-Palomino, R.; Oliveira-Filho, A.T. \& Pennington, R.T. 2010. Neotropical seasonally dry forests: Diversity, endemism, and biogeography of woody plants. In: Dirzo, R.; Young, H.S.; Mooney, H.A.; Ceballos, G. (eds.). Seasonally dry tropical forests: Ecology and conservation. Island Press, Washington, D.C. Pp. 3-21.

López, R.P.; Alcázar, D.L. \& Macía, M.J. 2006. The arid and dry plant formations of South America and floristic connections: new data, new interpretation? Darwiniana 44: 18-31.

Lucas, E.; Nunes, T. \& Lughadha, E.N. 2012. Lista preliminar da família Myrtaceae na região Nordeste do Brasil. Vol. 5. Kew Publishing, Royal Botanic Gardens, Kew. 38p.

Machado, I.C.S.; Barros L.M. \& Sampaio, E.V.S.B. 1997. Phenology of caatinga species at Serra Talhada, PE, Northeastern Brazil. Biotropica 29: 57-68. 
Machado, R.F. \& Queiroz, L.P. 2012. A new species of Prosopanche (Hydnoraceae) from northeastern Brazil. Phytotaxa 75: 58-64.

Machado, W.J.; Prata, A.P.N. \& Mello, A.A. 2012. Floristic composition in areas of Caatinga and Brejo de Altitude in Sergipe state, Brazil. Check List 8: 1089-1101.

Magurran, A.E. 2013. Medindo a diversidade biológica. Editora da UFPR, Curitiba. 261p.

Melo, F.P.; Siqueira, J.A.; Santos, B.A.; Alvares-da-Silva, O.; Ceballos, G. \& Bernard, E. 2014. Football and biodiversity conservation: FIFA and Brazil can still hit a green goal. Biotropica 46: 257-259.

Mendes, M.R.A. \& Castro, A.A.J.F. 2010. Vascular flora of semi-arid region, São José do Piauí, state of Piauí, Brazil. Check List 6: 39-44.

MMA. 2008. Lista oficial das espécies da flora brasileira ameaçadas de extinção. Disponível em <http:// www.mma.gov.br/estruturas/ascom_boletins/_ arquivos/83_19092008034949.pdf>. Acesso em 17 fevereiro 2013.

Moro, M.F.; Lughadha, E.N.; Filer, D.L.; Araújo, F.S. \& Martins, F.R. 2014. A catalogue of the vascular plants of the Caatinga Phytogeographical Domain: a synthesis of floristic and phytosociological surveys. Phytotaxa 160: 1-118.

Moro, M.F.; Araújo, F.S.; Rodal, M.J.N. \& Martins, F.R. 2015. Síntese dos estudos florísticos e fitossociológicos realizados no semiárido brasileiro (no prelo). In: Eisenlohr, P.V.; Felfili, J.M.; Melo, M.M.R.F. \& Meira Neto, J.A.A. Fitossociologia no Brasil: Métodos e estudos de caso. Vol. II. Editora da Universidade Federal de Viçosa, Viçosa.

Oliveira, G. \& Diniz-Filho, J.A.F. 2010. Spatial patterns of terrestrial vertebrates richness in Brazilian semiarid, Northeastern Brazil: selecting hypotheses and revealing constraints. Journal of Arid Environments 74: 1418-1426.

Oliveira-Filho, A.T.; Cardoso, D.; Schrire, B.D.; Lewis, G.P.; Pennington, R.T.; Brummer, T.J.; Rotella, J. \& Lavin, M. 2013. Stability structures tropical woody plant diversity more than seasonality: insights into the ecology of high legume-succulent-plant biodiversity. South African Journal of Botany 89: 42-57.

Oliveira-Filho, A.T.; Jarenkow, J.A. \& Rodal, M.J.N. 2006. Floristic relationships of seasonally dry forests of eastern South America based on tree species distribution patterns. In: Pennington, R.T.; Lewis, G.P. \& Ratter, J.A. (eds.). Neotropical savannas and dry forests: plant diversity, biogeography, and conservation. Taylor \& Francis CRC Press, Oxford. Pp. 59-192.

Pennington, R.T.; Lavin, M. \& Oliveira-Filho, A. 2009. Woody plant diversity, evolution, and ecology in the tropics: perspectives from Seasonally Dry Tropical Forests. Annual Review of Ecology, Evolution and Systematics 40: 437-57.
Pennington, R.T.; Prado, D.E. \& Pendry, C.A. 2000. Neotropical seasonally dry forests and Quaternary vegetation changes. Journal of Biogeography 27: 261-273.

Prado, D.E. \& Gibbs, P.E. 1993. Patterns of species distributions in the dry seasonal forests of South America. Annals of the Missouri Botanical Garden 80: 902-927.

Prado, D.E. 2000. Seasonally dry forests of tropical South America: from forgotten ecosystem to a new phytogeographic unit. Edinburgh Journal of Botany 57: 437-461.

Prado, D.E. 2003. As caatingas da América do Sul. In: Leal, I.R.; Tabarelli, M. \& Silva, J.M.C. (eds.). Ecologia e conservação da Caatinga. Editora Universitária, Universidade Federal de Pernambuco, Recife. Pp 3-74.

Queiroz, L.P. \& Cardoso, D.B.O.S. 2008. A new species of Aeschynomene L. (Leguminosae, Papilionoideae) from a continental sand dune area in north-eastern Brazil. Botanical Journal of the Linnean Society 157: 749-753.

Queiroz, L.P. \& Lavin, M. 2011. Coursetia (Leguminosae) from Eastern Brazil: nuclear ribosomal and chloroplast DNA sequence analysis reveal the monophyly of three Caatinga-inhabiting species. Systematic Botany 36: 69-79.

Queiroz, L.P. 2006. The Brazilian caatinga: Phytogeographical patterns inferred from distribution data of the Leguminosae. In: Pennington, R.T.; Lewis, G.P. \& Ratter, J.A. (eds.). Neotropical savannas and dry forests: plant diversity, biogeography, and conservation. Taylor \& Francis CRC Press, Oxford. Pp. 113-149.

Queiroz, L.P.; Conceição, A.A. \& Giulietti, A.M. 2006. Nordeste semi-árido: Caracterização geral e lista das fanerógamas. In: Giulietti, A.M.; Conceição, A.A. \& Queiroz, L.P. (eds.). Diversidade e caracterização das fanerógamas do semi-árido brasileiro. Vol.1. IMSEAR, MCT, Recife. Pp.15-359.

Queiroz, L.P.; Lewis, G.P. \& Wojciechowski, M.F. 2010. Tabaroa, a new genus of Leguminosae tribe Brongniartieae from Brazil. Kew Bulletin 65: 189-203.

Reis, A.M.S.; Araújo, E.L.; Ferraz, E.M.N. \& Moura, A.N. 2006. Inter-annual variations in the floristic and population structure of an herbaceous community of “caatinga” vegetation in Pernambuco, Brazil. Revista Brasileira de Botânica 29: 497-508.

Rocha, P.L.B.; Queiroz, L.P. \& Pirani, J.R. 2004. Plant species and habitat structure in a sand dune field in the Brazilian Caatinga: A homogenous habitat harbouring an endemic biota. Revista Brasileira de Botânica 27: 739-755.

Rodal, M.J.N. 1992. Fitossociologia da vegetação arbustivo-arbórea em quatro áreas de caatinga em Pernambuco. Tese de Doutorado. Universidade Estadual de Campinas, Campinas. 198p. 
Rodal, M.J.N. \& Nascimento, L.M. 2006. The arboreal component of a dry forest in northeastern Brazil. Brazilian Journal of Biology 66: 479-491.

Rodal, M.J.N.; Barbosa, M.R.V \& Thomas, W.W. 2008. Do the seasonal forests in northeastern Brazil represent a single floristic unit? Brazilian Journal of Biology 68: 467-475.

Roque, A. Queiroz, R.T. \& Loiola, M.I.B. 2009. Diversidade florística do Serido Potiguar. In: Freire, E.M.X. (org.). Recursos naturais das caatingas: uma visão multidisciplinar. Natal, EDUFRN. Pp. 11-49.

Sampaio, E.V.S.B.; Giulietti, A.M.; Virgínio, J. \& Gamarra-Rojas, C.F.L. (eds.). 2002. Vegetação e flora da caatinga. APNE/CNIP, Recife. 176p.

Santos, J.C.; Leal, I.R.; Almeida-Cortez, J.S.; Fernandes, G.W. \& Tabarelli, M. 2011. Caatinga: The scientific negligence experienced by a dry tropical forest. Tropical Conservation Science 4: 276-286.

Santos, J.M.F.F.; Santos, D.M.; Lopes, C.G.R.; Silva, K.A.; Sampaio, E.V.S.B. \& Araújo, E.L. 2013. Natural regeneration of the herbaceous community in a semiarid region in Northeastern Brasil. Environmental Monitoring and Assessment 185: 8287-8302.

Santos, R.M.; Oliveira-Filho, A.T.; Eisenlohr, P.V.; Queiroz, L.P.; Cardoso, D.B.O.S. \& Rodal, M.J.N. 2012. Identity and relationships of the Arboreal Caatinga among other floristic units of seasonally dry tropical forests (SDTFs) of north-eastern and Central Brazil. Ecology and Evolution 2: 409-428.

Särkinen, T.; Iganci, J.R.V.; Linares-Palomino, R.; Simon, M.F. \& Prado, D.E. 2011. Forgotten forests - issues and prospects in biome mapping using Seasonally Dry Tropical Forests as a case study. BMC Ecology 11: 27.
Sarmiento, G. 1975. The dry formations of South America and their floristic connections. Journal of Biogeography 2: 233-251.

SEI (Superintendência de Estudos Econômicos e Sociais do Estado da Bahia). 2009. Banco de dados geoambientais. Disponível em <http:// www.sei.ba.gov. br>. Acesso em 25 outubro 2009.

Silva, K.A.; Santos, J.M.F.F.; Santos, D.M.; Ferraz, E.M.N. \& Araújo, E.L. 2013. Spatial variation in the structure and composition of the herbaceous community in a semiarid region of northeastern Brazil. Brazilian Journal of Biology 73: 135-148.

Silva, R.F.S. \& Rapini, A. 2009. Allamanda calcicola (Apocynaceae), an overlooked new species from limestones in the States of Minas Gerais and Bahia, Brazil. Kew Bulletin 64: 171-174.

Tabarelli, M. \& Vicente, A. 2004. Conhecimento sobre plantas lenhosas da Caatinga: Lacunas geográficas e ecológicas. In: Silva, J.M.C.; Tabarelli, M.; Fonseca, M.T. \& Lins, L.V. (eds.). Biodiversidade da Caatinga: Áreas e ações prioritárias para a conservação. Ministério do Meio Ambiente, Brasília. Pp. 101-111.

Trejo, R.I. \& Dirzo, R. 2002. Floristic diversity of Mexican seasonally dry tropical forests. Biodiversity and Conservation 11: 2063-2048.

Tuomisto, H.; Ruokolainen, K. \& Yli-Halla, M. 2003. Dispersal, environment, and floristic variation of Western Amazonian forests. Science 299: 241-244.

Velloso, A.L.; Sampaio, E.V.S.B.; Giulietti, A.M.; Barbosa, M.R.V.; Castro, A.A.J.F.; Queiroz, L.P.; Fernandes, A.; Oren, D.C.; Cestaro, L.A.; Carvalho, A.J.E.; Pareyn, F.G.C.; Silva, F.B.R.; Miranda, E.E.; Keel, S. \& Gondim, R.S. 2002. Ecorregiões: Propostas para o Bioma Caatinga. APNE, The Nature Conservancy do Brasil, Recife. 76p. 
